ANUARIo DE Estudios MEDIEVAles (AEM)

37/1, enero-junio de 2007

pp. 71-106

ISSN 0066-5061

\title{
IL TESORO RECUPERATO. \\ L'INVENTARIO DEI BENI DELLE REGINE DI SICILIA CONFISCATI A MANFREDI ALAGONA NEL $1393^{1}$
}

\author{
THE RECOVERED TREASURE. \\ THE INVENTORY OF THE PROPERTY OF SICILIAN QUEENS \\ CONFISCATED BY MANFREDI ALAGONA IN 1393
}

\author{
DANIELA SANTORO \\ Università di Palermo
}

Riassunto: Partendo dal ritrovamento, presso l'Archivio di Stato di Palermo, di un inventario di beni confiscati a Manfredi Alagona nel 1393, il lavoro ricostruisce il percorso del tesoro delle regine siciliane (Costanza d'Aragona, Maria di Sicilia) che era stato incamerato dagli Alagona, potente famiglia di origine aragonese trapiantata in Sicilia. Uno studio dettagliato degli oggetti inventariati permette l'analisi di vari aspetti legati al costume e alla società sul finire del XIV secolo, dall'abbigliamento alla tavola, dal gusto e la moda del tempo alla cura della persona, dalla passione per i gioielli al culto per le reliquie.

Parole chiave: inventario, regine, tesoro, gioielli, costume, arredamento, reliquie.

\begin{abstract}
Beginning from the discovery, in the "Archivio di Stato" of Palermo, of an inventory containing the belongings confiscated from Manfredi Alagona in 1393, this work reconstructs the story of the treasure of the Sicilian queens (Costanza of Aragon and Maria of Sicily), that had been confiscated from the powerful Alagona family, originally from Aragon, who moved to Sicily. A detailed study of the inventoried movables allows the analysis of several aspects connected to customs and society at the end of the XIV ${ }^{\text {th }}$ century, from clothing to utensils, from taste and fashion to care of appearance, from the love of jewels to the workship of relics.
\end{abstract}

Keywords: inventory, queens, treasure, jewels, customs, movables, relics.

${ }^{1}$ Abbreviazioni utilizzate: Acta Curie: Acta Curie Felicis Urbis Panormi; ADM: Archivio General de la Fundación Casa Ducal de Medinaceli- ASP. Archivio di Stato di Palermo; ASPN: "Archivio Storico per le Province Napoletane"; ASS: "Archivio Storico Siciliano"; ASSO: "Archivio Storico per la Sicilia Orientale"; BCP: Biblioteca Comunale di Palermo; DBI: "Dizionario biografico degli Italiani"; DSSS: "Documenti per servire alla storia di Sicilia"; Misc. Arch.: Miscellanea Archivistica; Protonot.: Protonotaro del Regno (fondo dell'Archivio di Stato di Palermo); $R$. Canc.: Real Cancelleria (fondo dell'Archivio di Stato di Palermo); Tabulario di S. Maria Maddalena: Tabulario di S. Marian

Desidero ringraziare i professori Laura Sciascia, Salvatore Fodale, per avere incoraggiato e seguito da vicino la stesura di questo lavoro. Ringrazio inoltre la professoressa Patrizia Sădina che mi ha aiutato nella traduzione dall'italiano all'inglese. 


\section{SOMMARIO}

1. Passaggi. - 2. Dall'Aragona alla Sicilia: la dote di Costanza d'Aragona. - 3. Gioielli. - 4. Vesti e accessori. - 5. Il corredo. - 6. La tavola. - 7. Arredi e ornamenti. - 8. Altari e celebrazioni. - Appendice.

\section{PASSAGGI}

Il 16 novembre 1392 Martino il Vecchio scriveva, a doge e comune di Genova, a proposito di certi gioielli que pertinebant ad reginam Mariam. Era infatti venuto a conoscenza del fatto che Artale Alagona, proditor manifestissimus et rebellis, bisognoso di procurarsi aiuti per proseguire la sua resistenza all'affermazione dei Martini in Sicilia, aveva inviato a Genova una considerevole quantità di oggetti preziosi, impegnandoli: una croce aurea coronarum et nonnulla vasa argentea et iocalia in numero et pondere satis grandi, in mano di Artale per tempora satis longa che appartenevano, sottolineava Martino, serenissime domine regine Sicilie nostre sorori, alla sorellastra Costanza d'Aragona, figlia di Pietro IV e della prima moglie, Maria di Navarra. Al doge genovese, Martino chiedeva la restituzione di quegli oggetti che, a die obitus antedicte regine, erano stati custoditi dagli Alagona nel castello di Aci: la corona d'oro, i vasellami d'argento, i gioielli spettavano pleno iure alla regina di Sicilia Maria ut universali eredi della madre Costanza, al marito di Maria, Martino e, puntualizzava Martino il Vecchio, nobis nomine eorundem ${ }^{2}$. Figlio dell'omonimo duca di Montblanc, il secondogenito del re d'Aragona, Martino il Giovane, più piccolo di Maria di quattordici anni, aveva sposato l'erede al trono isolano, figlia di Costanza d'Aragona e Federico IV di Sicilia ${ }^{3}$.

Il filo rosso degli intrecci tra le case regnanti siciliana e aragonese si snodava indietro nei secoli: a partire dal 1080 , puntualmente scanditi, una serie di rapporti politico-matrimoniali tra Barcellona, Aragona, normanni d'Italia, aveva contribuito a tessere un legame ora sommerso ora evidente, "ponti" gettati, scrive Francesco Giunta, "tra le due sponde del Mediterraneo" ${ }^{4}$ : fin dal 1262 anno di un matrimonio -destinato a scuotere il Mediterra-

${ }^{2} \mathrm{BCP}, \mathrm{ms} . \mathrm{QqG5}$, cc. 378 v -379. Cfr. Isidoro LA LuMIA, Storie siciliane, a c. di. Francesco GIUNTA, II Palermo 1969 , p. 252 e Salvatore FODALE, Le reliquie del re Martino, in "Aspetti e momenti di storia della Sicilia (Secc. IX-XIX). Studi in memoria di Alberto Boscolo", Palermo 1989 , pp. 125 s. Sulla situazione isolana al momento dell'arrivo dei Martini nell'isola, Francesco GIUNTA, Aragonesi e catalani nel Mediterraneo, I, Dal regno al viceregno in Sicilia, Palermo 1953, pp. 188 ss.

${ }^{3}$ Rafael TAsIS I MARCA, Pere el Cerimoniós i els seus fills, Barcelona, 1962, p. 107; Maria Teresa FERRER I MALLOL, Maria I de Sicilia, in Gran Enciclopèdia Catalana, Barcelona, 1976, vol. 9 , p. 600 .

${ }^{4}$ Francesco GiunTA, Geronimo Zurita e il regno normanno svevo di Sicilia, in Non solo Medioevo. Dal mondo antico al contemporaneo, Palermo, 1991, p. 120. 
neo ${ }^{5}$ - tra l'erede di Manfredi di Svevia, Costanza, e Pietro infante di Catalogna, il futuro Pietro III el $\mathrm{Gran}^{6}$.

Passaggio dietro passaggio, arrivato nell'isola dalla Catalogna assieme a Costanza, la sposa di Federico IV, un vero e proprio tesoro di perle, pietre preziose, magnifici capolavori di oreficeria, raffinati manufatti, era finito in buona parte tra le mani di una famiglia aragonese, gli Alagona, trasferitasi nella parte finale del XIII secolo: assieme ad altri capostipiti di famiglie siculo-aragonesi, Blasco Alagona il Vecchio era venuto in Sicilia, ha scritto Laura Sciascia, "nel momento in cui maggiormente l'isola si chiude al resto del mondo", dopo il Vespro, fermandosi poi come esule ${ }^{7}$. Lasciato da fratello Artale "por cabeza y pariente mayor de su casa" 8 , Manfredi Alagona, rector et gubernator in regimine dicti fratris sui ${ }^{9}$, in virtù delle benemerenze acquisite nei confronti della Corona, otteneva da Pietro IV d'Aragona la conferma nel 1378, un anno dopo la morte di Federico IV, di quanto ereditato sino a quel momento ed accumulato per donacionem dei sovrani ${ }^{10}$.

Nel 1390 Manfredi diventava gran giustiziere del regno di Sicilia (ad Artale, Federico IV il 18 gennaio 1363 aveva riconosciuto l'ereditarietà della carica) e, gestendo assieme ai fratelli la maggior parte del regno, controllava una grossa fetta di territorio isolano, Messina con la piana di Milazzo,

${ }^{5}$ David ABULAFIA, I regni del Mediterraneo occidentale dal 1200 al 1500 . La lotta per il dominio, Roma-Bari, 1999, $\mathrm{p}_{\text {. }}$ 27. Cfr "Helene WIERUSZOWSKI, La corte di Pietro d'Aragona e pp. 200-217; Alberto BosCOLO, L'eredità sveva di Pietro il Grande, re d'Aragona, in Catalani nel Medioevo, Bologna, 1986, pp. 73-88.

${ }^{6} \mathrm{Cfr}$. Daniel Girona Llagostera, Mullerament del Infant En Pere de Cathalunya ad Madona Costança de Sicilia, Barcelona, 1909.

${ }^{7}$ Laura SCIASCIA, Le donne e $i$ cavalier, gli affanni e gli agi, Messina, 1993, p. 18. Dal Vespro in poi molti nobili catalani si recavano nell'isola al servizio di Pietro III, alcuni stabilendosi definitivamente, Maria-Mercè COSTA, Les families catalans a Sicilia, in Els catalans, pp 73-80 e Maria Teresa FERRER I MALLOL, Nobles catalans arrelats a Sicília: Guillelm Ramon 1 de Montcada, in "Mediterraneo medievale. Scritti in onore di F. Giunta", I, Soveria Mannelli, 1989 , pp. $417-432$.

${ }^{8}$ Jeronimo ZuriTA, Anales de la Corona de Aragón, a c. di A. Canellas Lopez, 4, p. 758. Nel 1355 Artale Alagona aveva ereditato dal padre Blasco il Giovane (nipote di Blasco il Vecchio) l'ufficio di gran giustiziere e la contea di Mistretta, GIUNTA, Alagona, Artale, in DBI, 1, Roma 1960, p. 556. A Maria, avuta dall'ultima moglie Agata nel codicillo del 5 febbraio 1388 aggiunto al testamento - Artale destinava una dote di ottomila onze se si fosse sposata extra insolam commorante, cinquemila in pecunia numerata, mille in jogalibus et arnesio. Artale disponeva po che tot arma vel pavisios, coracias, crivelleras, lanceas, dardos, bellicones precii decem millium florenorum sufficentia pro munimentis fossero conservati nel palazzo di Messina e nel castello di Aci, e specificava: in cammara nostra habere tantam pecuniarum quantitatem ex qua satisfacio presentis testamenti comode fieri poterit et in precium cuius numerus non expedit reservare, Isidoro LA LUMIA, Estratti di un processo per lite feudale de secolo XV, in DSSS, I s., Diplomatica, III, r.a. Palermo, 1990, pp. 190 ss.

${ }^{9}$ LA LUMIA, Estratti, p. 21. Il 26 marzo 1389, il duca rispondeva a una lettera di Maria per la qual me certificats de la mort de don Artal d'Alagón, BCP., ms. QqG5, c. 33 v., avvenuta nel febbraio, Francesco GIUNTA, Alagona, Artale, p. 557.

${ }^{10}$ Antonino GIUfFridA, Il cartulario della famiglia Alagona di Sicilia. Documenti 1337-1387, in Acta siculo-aragonensia, I, Palermo, 1978, p. 93, doc. XCIII (7.7.1378). A 36 anni, il 27 in Acta siculo-aragonensia, I, Palermo, 1978, p. 93, doc. XClil Federico IV muore a Messina per una malattia all'intestino: dissenteria o cancro o forse, girava voce, avvelenato da Artale per una malattia all intestino: dissenteria o cancro o torse, girava voce, avvelenato da Artale nozze del re Federico III con la principessa Antonia del Balzo, Palermo, 1895, pp. 70 ss.; LA

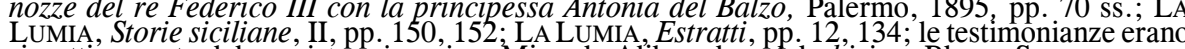
rispettivamente del magister cirurgicus Mino de Alibrando e del phisicus Blasco Scammacca. 
Taormina, Francavilla, Catania, La Motta, Calatabiano, Noto, Randazzo ${ }^{11}$. Ottenuto da Bonifacio IX il riconoscimento ufficiale della carica di vicario, il 10 luglio 1391, nella chiesa di San Pietro "que estaba en el campo en el territorio de Castronuevo junto al río", Manfredi aveva fatto giuramento assieme ad Andrea Chiaromonte, Antonio Ventimiglia e Guglielmo Peralta - i quattro vicari "o por decirlo más propriamente, cuatro príncipes y señores de aquella isla, de quien pendía todo el gobierno de la paz y de la guerra" - di non ricevere il duca "si no fuesen todos en ello concordes y de un ánimo" Tre mesi dopo l'incontro di Castronovo, il 23 ottobre 1391 Martino scriveva al vescovo di Tortosa: E com nos ara com som stats aci en Barchinona haiam trobada una galea en la qual son venguts a la reyna de Sicilia et a nos missatgers de Manfre d'Alago et de Andriota de Claramunt et de moltes ciutats offrendosi di ricevere i nuovi sovrani com a Senyors ${ }^{13}$.

Dalla Sicilia erano dunque giunti messaggeri di Andrea Chiaromonte e Manfredi Alagona a la reyna de Sicilia et a nos, scriveva Martino: la galea con i messaggeri di Andrea era tornata indietro a Favignana, i messaggeri di Manfredi no sen volgueren tornar, ans son venguts ab la dicta galea segons dit es los quals an portades diverses joyes, les quals an presentades de part del dit Manfre a la dicta reyna, et de part da quell nos an supplicat humilment los deguessem provehir dalguns officis en la dicta illa ${ }^{14}$. Schiusi i forzieri, Manfredi aveva offerto gioielli segno della sua disponibilità a riconoscere Maria come regina.

A picco sul mare, nel castello di Aci -super ingenti saxo fundatum $^{15}$ - erano stati in buona parte accumulati l'oro e i preziosi oggetti appartenuti ai re e alle regine di Sicilia, soprattutto Costanza e Maria, a partire dal primo Artale tutore, sulla base delle disposizioni testamentarie di Federico IV, della figlia del re Maria ${ }^{16}$ sfuggito alla cattura il 18 maggio 1392, quando in carcere erano finiti Manfredi con il figlio Iayme e Andrea

\footnotetext{
${ }^{11}$ Francesco GIUnTA, Alagona, Manfredi, in DBI,1, Roma, 1960, pp. 559 s.; Fodale, Federico IV, p. 698.

${ }^{12}$ ZURITA, Anales, 4, pp. 759 s. Sulla "signoria meridionale" dei Peralta, Maria Antonietta Russo, I Peralta e il Val di Mazara nel XIV e XV secolo, Caltanissetta-Roma, 2003.

${ }^{13}$ ASP, Protonot., reg. 6, cc. 77 v.-79 (23.10.1391) e GIUNTA, Aragonesi, p. 192, nota 6.

${ }^{14}$ ASP, Protonot $_{\text {. }}$ reg. 6, c. 83 (27.10.1391) e BCP., ms. QqG5, cc. 46 v.-47. Il primo giugno 1392 , il duca Martino in una lettera al re d'Aragona per informarlo della cattura di Andrea e della reductione di Palermo ad regiam fidelitatem, a un certo punto scriveva che, fatta richiesta Andrea di un colloquio con il duca, assieme all'arcivescovo di Palermo, volents se sculpar da co que mal havyen fevt en temps passat, digueren contra ells mateix molts coses donant carrech Manfre d'Alago, BCP, ms. OgG5, cc. $211 \mathrm{v} .-215 \mathrm{r}$. Attribuire a Manfredi la colpa di quella tattica temporegoiatrice e, alla fine, apertamente contraria all'insediamento dei Martini nell' '1sola non valeva a salvare la vita di Andrea quello stesso primo di giugno decapitato nella pisozza non valeva a saivare la vita di Andrea, quello stesso primo di giugno decapitato nella piazza antistante il palazzo di famiglia, o Steri. E la notizia di uno steri che Artale Alagona possedeva a Palermo aveva preoccupato Andrea Chiaromonte che, all'inizio di gennaio del 1392, ne scriveva al suocero e pretore Federico Cisario, Acta Curie felicis urbis Panormi, vol. 10, Registri di lettere

${ }_{15}^{15}$ Nicolò SPECIALE, Historia sicula, in Rosario GREGORIO, Bibliotheca scriptorum qui res in Sicilia gestas sub Aragonum imperio retulere, Palermo, 1791, I, p. 381.

${ }^{16}$ Sul testamento di Federico, Salvatore Fodale, Federico IV, in DBI, 45, Roma, 1995, p.
} 700 . 
Chiaromonte $^{17}$, appresa la notizia della prigionia del padre -riferiva il duca in una lettera del 20 luglio 1392 al fratello Giovanni re d'Aragona- Artale se mes en lo castell appellat Iachi, qui es prop de la ciutat de Cathania molt fort e quasi inexpugnable, castello nel quale si trovavano tots les joyes, or et argent de la reyna e tot lo bon moble del dit Manfre ${ }^{18}$.

L'ordine di confisca dei beni di Manfredi Alagona - che il duca sapeva essere conservati ad Aci- era partito il giorno della cattura di Manfredi. Quello stesso 18 maggio 1392, dall'assedio di Palermo, Martino aveva indirizzato una lettera ai catanesi con l'ordine perentorio di catturare expresse e arrestare Artale Alagona, facendo inventariare i beni - pecunias, iocalia, suppellectilia et utensilia ac omnia et singula alia bona mobilia seu immobilia - appartenuti a Manfredi e ai suoi figli. Inventariati, quei beni avrebbero dovuto essere affidati al mercante barcellonese Guglielmo Ça Trilla il quale, caricatili a bordo della sua nave, avrebbe dovuto consegnarli a un funzionario e uomo di fiducia del duca ${ }^{19}$. Allo stesso Ca Trilla ritornato in Catalogna, il duca scriveva mentre si trovava a Catania - Artale Alagona protagonista, nel marzo 1394, di un secondo ingresso in città- a proposito di un tabernacle on es la image di la Verge Maria, che il mercante avrebbe dovuto consegnare a un uomo di fiducia di Martino ${ }^{20}$.

Da quel 18 maggio 1392, erano passati nove mesi che Manfredi Alagona aveva trascorso in carcere; latitante e asserragliato, in perenne evoluzione d'intenti rispetto alla possibilità di arrendersi o no, il figlio Artale mittente e destinatario di una messe di lettere e messaggi con il duca Martino numerosa al punto, notava Guardione, da potere costituire un Codice diplomatico ${ }^{21}$.

\footnotetext{
${ }^{17}$ Cfr. LA Lumia, Storie siciliane, II, pp. 229 ss. Sul giovane Artale, Salvatore FodALE, Il conte e il segretario. L'ultimo Artale d'Alagona e il giurista Stefano Migliarisi: due storie incrociate, in "Mediterraneo medievale", I, pp. 442 ss.

${ }^{18}$ BCP, ms. QqG5, cc. 289-292 r. Concesso da Federico III a Blasco Alagona conte di Mistretta che lo aveva tenuto per circa un ventennio, scambiandolo poi per un altro castello, Aci era in seguito stato donato da Federico IV ad Artale, concessione ratificata da Simone del Pozzo (Aci infatti era considerato da parte ecclesiastica proprietà della chiesa di Catania); nel 1380 Urbano VI aveva ratificato la concessione, nel 1391 papa Bonifacio IX assegnava castrum et terram Iacii a Manfredi Alagona, Salvatore FODALE, Scisma ecclesiastico e potere regio in Sicilia, I, Il Duca di Montblance l'episcopato tra Roma e Avignone (1392-1396), Palermo, 1979 , pp. 12 s. e 172, doc. XXII del 4 luglio 1391.

${ }^{19} \mathrm{BCP}, \mathrm{ms}$. QqG5, c. 157. Notizie scarne sulla famiglia: Francesco Trilla era capitano di una galea di Barcellona, Santa Clara, inviata nel 1390 contro i ribelli sardi, Geraldo Trilla cittadino barcellonese nel 1302 si occupava di spedire una provvista di cannella da Barcellona a Montpellier (Antonio de CAPMANY Y DE MONPALAU, Memorias históricas sobre la marina, commercio y artes de la antigua ciudad de Barcelona. Barcelona, 1961-1963, I, p. 190; II, 1 , p. 360 e II 1 p. 99) Berengario Ca Trilla era domicellus dei Martini cui si rivolgeva per riavere 1 centoventi fiorini dovutigli dal miles Guglielmo Cartellano, ASP, R. Canc., reg. 18, c. 61 (13.9.1393)

${ }^{20}$ ASP, Protonot., reg. 5, c. 300 r: (28.06.1394). Sul secondo assedio di Aci, ZURITA, Anales, 4, pp. 774 ss.; LA LUMIA, Storie III, pp. 280 ss. Sui privilegi dei catalani in Sicilia Mario DEL TREPPO, I mercanti catalani e l'espansione della Corona d'Aragona nel secolo XV Napoli 1972, pp. 149 ss. Sulla "traiettoria" mediterranea e lo spazio dei mercanti catalani dal Vespro in poi, dello stesso DEL TREPPO, L'espansione catalano-aragonese nel Mediterrano, in "Nuove questioni di storia medioevale", Milano, 1964, pp. 262 ss.

${ }^{21}$ Francesco GUARDIONE, Documenti sul secondo assedio di Catania e suo riordinamento del Regno di Sicilia (1394-96), in ASSO, I, 1904, pp. 81-83.
} 
Nove mesi dopo l'emissione del mandato, nel febbraio 1393, Maria e i Martini ricevevano da Ça Trilla l'apocha attestante la consegna a Guglielmo Serra - camerario della regina, che si era occupato di stilare l'inventario- dei beni que fuerunt Manfridi de Alagona ${ }^{22}$.

Quei beni comprendevano, in realtà, una parte del tesoro delle regine di Sicilia, tesoro che confluito tra i beni di famiglia degli Alagona era stato custodito nel castello di Aci, fino alla confisca: emblematico il fatto che nessuno degli oggetti inventariati - abiti, cinture, cappelli, spettacolari pezzi di oreficeria, coperte, lenzuola, servizi da tavola, soprammobili, l'occorrente per arredare un palazzo - rechi lo stemma di famiglia degli Alagona, arme d'oro "a sei torte di nero, ordinate, 2,2 e 2 " 23 .

\section{DALl'Aragona Alla Sicilia: LA DOTE DI COSTANZA D'ARAGONA}

$$
\begin{array}{r}
\text { A veces he sentido remordimiento } \\
\text { y otras envidia, } \\
\text { de ti que estás, como nosotros, en el tiempo y su laberinto } \\
\text { y que no lo sabes. } \\
\text { J.L. BoRGES, } A \text { una moneda }
\end{array}
$$

Maria di Navarra. Costanza d'Aragona. Maria di Sicilia. Nonna, figlia, nipote, impalpabili aleggiano sullo sfondo di questa storia legate da un filo, visibile appena, che intreccia perle, gioielli, smalti: muti testimoni dei passaggi di potere, della capacità insita nelle cose di rivelare, evocare, trascinare. Della brama di possederle, le cose.

Maria di Navarra, nata nel 1322, era scomparsa venticinquenne appena. Figlia di Filippo, re di Navarra e conte d'Évreux - che aveva sposato Giovanna, regina di Navarra e contessa di Champagne- era diventata la moglie di Pietro IV d'Aragona nel 1338. Messi al mondo cinque figli, delle tre femmine Costanza, nata nel 1340 a Poblet, nel 1347 era stata proclamata erede della Corona catalano-aragonese, fatto insolito che aveva causato le proteste del fratello di Pietro, Jaume d'Urgell, contrasto risolto a seguito della nascita nel 1350 di Giovanni I, l'erede maschio che a Pietro aveva dato la terza moglie, la siciliana Eleonora ${ }^{24}$.

Tra i figli, maschi e femmine, di Pietro IV, Costanza sembra fosse "un dels fills que més estimà el rei, el qual, en no poderl-la fer successora directa seva, va destinare-li un altre ofici insigne: convertida en reina de Sicília, havia d'ésser l'artífex de la incorporació del regne insular a la Corona

\footnotetext{
${ }^{22} \mathrm{Cfr}$. inventario in Appendice.

${ }^{23}$ Antonio MANGO DI CASALGERARDO, Nobiliario di Sicilia, Bologna, 1970, r.a. dell'edizione di Palermo, 1912-1915, I, 47 e tavola Vì.

${ }^{24}$ Un profilo di Maria di Navarra e di Costanza d'Aragona in Gran Enciclopèdia Catalana vol. 9, p. 603 e vol. 5 , pp. 525 s. Su Jaume I d'Urgell e Filippo di Navarra, ivi, vol. 8, p. 736 e vol. 7 , p. 347. Cfr. ZuRiTA, Anales, 4, pp. 23 ss. 
d'Aragó" 25 . Non riuscito il progettato matrimonio con Ludovico di Sicilia scomparso nell'ottobre 1355, candidato a sposare Costanza era il fratello e successore di Ludovico, Federico IV, cugino e cognato al contempo di Pietro $\mathrm{IV}^{26}$.

Al momento delle nozze con Federico IV, Costanza aveva ventidue anni, tre più "que el germà de la seva madrastra que li era destinat com a marit" ${ }^{27}$. Breve il suo soggiorno siciliano: data alla luce una figlia, Maria, a Catania, nel $1362^{28}$, nel luglio 1363 Costanza si ammalava, prisa di una pestifera ed acerba febri, di forma tali, che in brevi migrau di quista presenti vita a lo Signur ${ }^{29}$. Moriva a 24 anni, "deixant com a garantia de la realització del pla traçat pel seu pare i la seva madrastra, una filla" ${ }^{30}$ : la figlia attorno alla quale Federico costruiva il progetto di dare continuità al suo regno, escludendo l'Aragona e le sollecitazioni pressanti della sorella Eleonora ${ }^{31}$.

Gli splendidi manufatti di fattura catalana, navarrese, aragonese di chiara appartenenza regale - ereditati da Costanza d'Aragona dalla madre Maria di Navarra, trasmessi de iure alla figlia Maria di Sicilia che aveva sposato Martino il Giovane - finiti agli Alagona, erano reclamati adesso da Martino il Vecchio in virtù di diritti che su più piani ne rendevano legittima la restituzione.

Arrivato l'ordine di confisca, il camerario della regina Guglielmo Serra aveva redatto l'inventario dei beni "appartenuti" a Manfredi Alagona. Alcuni tra quei beni, dai capi d'abbigliamento ai pezzi di arredamento, inequivocabilmente erano appartenuti ai re d'Aragona, a Costanza per tre anni considerata l'erede della Corona aragonese-catalana: capo da cerimonia dei re aragonesi il mantum di velluto verde con merlettature dorate, cum frixo de auro ad arma de Aragona. E regali dovevano essere alcuni dei mantelli verdi e rosso porpora, i due colori preferiti dalle classi alte ${ }^{32}$ : un palium de diaspro e uno di zendado rosso foderati di tela verde, uno di panno dorato foderato di tela celeste, un pecium di un pallio di panno dorato.

Ad arma de Aragona: era di Costanza d'Aragona il cassone ad arma de Aragona - cassoni che servivano di solito a racchiudere il corredo nuziale

\footnotetext{
${ }^{25}$ TASIS I MARCA, Pere el Cerimoniós, p. 141.

${ }^{26}$ Sulla politica di Pietro Maria Teresa FERRER I MALlol, La politica siciliana de Pere el Cerimoniós, in Els catalans a Sicília, a c. di Francesco GIUNTA, M. DE RIQUER, J.M ${ }^{\mathrm{a}}$. SANS I TRAVÉ, Barcelona, 1992, pp. 35 ss.

${ }^{27}$ TASIS I MARCA, Pere el Cerimoniós, p. 99.

${ }^{28}$ Gran Enciclopèdia Catalana, vol. 5, pp. $525 \mathrm{~s}$.

${ }^{29}$ Anonimo, Historia sicula, in GREGORIO, Bibliotheca scriptorum, II, p. 296.

${ }^{30}$ TASIS I MARCA, Pere el Cerimoniós, p. 141.

${ }^{31}$ Da Perpignano, l'8 aprile 1356, Eleonora aveva scritto al protonotaro di Sicilia Perrono de Iuvenio ricordando i suoi diritti alla corona nel caso di morte di Federico senza prole, Emile G.LÉONARD, Histoire de Jeanne $I^{r}$ reine de Naples comtesse de Provence (1343-1382), III Monaco Paris, 1936 , pp. $600 \mathrm{~s}$., doc. LXIII. Cosa che continuava a fare nel corso degli anni, ne 1358 allorché dava istruzioni al segretario Berenguer Carbonell perchè sollecitasse Federico a fare donazione del regno a nos o a un de nostres fills en cas que ell moris sens fills, ivi, p. 631, doc. LXXXVIII; nel 1362, Federico sposato con Costanza, esercitava pressioni per la trasmissione della carta de donacio del regno, ivi, p. 663, doc. CXIV.

${ }^{32}$ Salvatore Tramontana, Vestirsi e travestirsi in Sicilia, Palermo, 1993, p. 95.
} 
e recavano motivi iconografici legati alla vita femminile ${ }^{33}-$, di Costanza il candelabro d'argento dorato fractum ad modum cope, ad arma de Aragona e un pecium de here ad arma Aragonum, marchiati con l'inconfondibile scudo bicolore, oro e rosso, catalano-aragonese.

Con gli stemmi di Navarra e Évreux, i gigli francesi e le catene navarresi, è lo scudo di Maria di Navarra, nella cappella di Sant'Agata a Barcellona $^{34}$, in quattro parti l'arme nel Nobiliario de la Corona de Aragón, prima e quarta catalano-aragonese, seconda di Navarra, terza di Évreux: sfondo azzurro e gigli d'oro "cargado de una cotiza de plata y gules" 35 , arricchito di una striscia trasversale in rosso e argento. Armi navarresi che si ritrovano in alcuni oggetti passati - attraverso Maria di Navarra, terza donnasfondo di questa storia- a Costanza, quindi alla figlia Maria di Sicilia: i cuscini di seta e velluto, di velluto e oro, ad arma de Navarra; una piccola saliera d'argento ad arma de Navarra; un paramurum di panno dorato e velluto carmisino cum ferciis decem septem foderato con panno serico verde, ad arma de Navarra.

Uno stemma tripartito - Sicilia, Aragona, Navarra- simbolo del legame tra le case regnanti, era impresso su una scalam d'argento con il suo astuccio $^{36}$, anche questa dunque, appartenuta a Costanza d'Aragona: come quelle date in pegno da Martino a Ximenes de Lerda nel novembre 1393, una scalam d'argento con sei esmalts, quattro con signa regali Aragonie, due con signa del re di Navarra, deaurata et stillata a parte superiori et marchata col marchio di Valenza; un'altra scalam d'argento totam deauratam et stillatam con sei smalti, due cum signo regali Aragonie, due cum signo domini regis Sicilie, le restanti due cum signo civitatis Barchinone $e^{37}$.

Possibile si trattasse di staffe ${ }^{38}$, ricchissimi erano, d'altronde, gli arredi utilizzati per cavalcare, dalle selle ai freni alle staffe, di panno dorato, finissimi ricami, d'oro e argento, spesso provenienti dalla Spagna a testimoniare, sottolineava Lanza di Scalea, "lo sfarzo signorile della nazione dominatrice" ${ }^{39}$. Nel 1382, Pietro IV aveva fatto modificare una sella che gli

\footnotetext{
${ }^{33}$ Ettore GABRICI-Ezio LeVI, Lo Steri di Palermo e le sue pitture, Milano-Roma, 1932, r.a. Palermo, 2003, pp. $210 \mathrm{~s}$.

${ }^{34}$ Gran Enciclopèdia Catalana, vol. 9, p. 603.

${ }^{35}$ Nobiliario de la Corona de Aragón, I, p. 144.

${ }^{36}$ Item scalam unam de argento ad arma Sicilie, de Aragona et de Navarra cum sua investa, cfr. inventario in Appendice. Simile allo stemma di Bianca di Navarra, la figlia di Carlo IIII sposa di Martino alla morte di Maria: sul lato sinistro Sicilia-Aragona, sul destro Navarra-Evreux Nobiliario de la Corona de Aragón, II, p. 37. Sulla Navarra e le linee politiche adottate, Jose María LACARRA, Historia del reino de Navarra desde sus origines hasta la Baja Edad Media, Pamplona ,1973., III. Su Bianca e la sua vita politica, Eloisa RAMÍREZ VAQUERO, La reina Blanca y Navarra, in "Príncipe de Viana", LX n. 217 (1999), pp. 323 ss. Sulla politica siciliana di Bianca, Salvatore FODALE, Blanca de Navarra y el gobierno de Sicilia, ivi, pp. 311 ss.

${ }^{37}$ ASP, $R$. Canc., reg. 18, cc. 101-102 r.; BCP, ms. QqG5, cc. 638-541 (21.11.1393).

${ }^{38}$ Charles Du Fresne Du CANGE, Glossarium mediae et infime latinitatis, Graz, 1954, ad vocem $\mathrm{n} .10$.

${ }^{39}$ Pietro LANZA DE SCALEA, Donne e gioielli in Sicilia nel Medioevo e nel Rinascimento, Palermo-Torino, 1892, pp. 39, $135 \mathrm{~s}$. Esperti cavalieri i re normanni e svevi, Salvatore TRAMONTANA, Il Regno di Sicilia. Uomo e natura dall'XI al XIII secolo, Torino, 1999, pp. 276 SS. 
era stata portata da Barcellona, chiedendo di apporvi il suo scudo e altre piccole correzioni che l'avrebbero resa più adatta ad un uomo: esta tot a nostra guisa, exceptat que en los esmalts del fre en torn los quals ha rosetes, volem que $n$ sien levades les dites rosetes e que si faca altra obratge ${ }^{40}$. Nel 1336 il miles Federico de Aragona aveva dato in pegno per una somma di 60 onze mutuata, una faluca per cavalcare furnitam de argento, con pietre preziose, smalti e perle lavorate ad arma di Federico e di Vinciguerra Palizzi, un freno di filo d'argento cum capenaglis et abenis, briglie, con pietre preziose, due staffe di filo d'argento, un pettorale di filo d'argento con smalti ad arma di Federico e dei Palizzi ${ }^{41}$.

Tra i beni inventariati confiscati a Manfredi, due sambucas d'argento cum frenis, selle apposite da donna ${ }^{42}$ che potevano essere decorate con materiali preziosi come oro, perle, argento, e intessute di sciamito, panno dorato, seta ${ }^{43}$.

\section{GIOIELLI}

Grande parte dei beni inventariati è costituita da gioielli. Da portare al collo un agnus Dei d'argento dorato appeso a un cordoncino di seta carmisina, un altro smaltato cum una cruceta de argento, un peciolum d'argento con un cordoncino di seta verde e una muscam d'argento dorato con pietre e perle ${ }^{44}$ : in occasione delle nozze con Federico III d'Aragona, Giovanni Maletta aveva offerto a Eleonora d'Angiò una grande muscam con un cammeo albo posito in medio in forma medie domine con pietre preziose e perle ${ }^{45}$. Manfredi aveva pure una canacca -voce araba per collana, diffusa nel dialetto siciliano ${ }^{46}$ - a dieci fili con quattro zaffiri.

Sorta di orecchini pendenti gemmati e circolari, cerchi d'oro con pietre preziose e perle, erano i circelli o auriculari poco diffusi in Europa e soprattutto in Francia, forse per la pettinatura che copriva le orecchie, diffusissimi nella Sicilia del XIV secolo, per l'influenza delle mode greca e

${ }^{40}$ Amada LóPez de Meneses, Documentos culturales de Pedro el Cerimonioso, Zaragoza 1952 , p. 97. ${ }^{41}$ Ferdinando LIONTI, Le società dei Bardi, dei Peruzzi e degli Acciainoli in Sicilia, in ASS,
n.s. XIV (1889), p. 193 e doc. II, 204.

${ }^{42}$ LANZA DI SCALEA, "Donne e gioielli, p. 122. Secondo la definizione di DU CANGE, Glossarium, ad vocem, "sambuca" era genus cynthurae rusticae. Sull'equipaggiamento femminile, Martí DE RIQUER, L'arnès del cavaller. Armes $i$ armadures catalanes medievals, Barcelona, 1968, pp. 138 ss.

${ }^{43}$ Ornamenti vietati da Federico III nei capitoli del 1308, Francesco TeSTA, Capitula regni Siciliae, I, Palermo, 1741, p. 90, cap. XCII.

${ }^{44} \mathrm{Cfr}$. inventario in Appendice.

${ }^{45}$ LanZa DI SCAlEa, Donne e gioielli, p. 141. Cfr. voce musca in Du CANGE, Glossarium.

${ }^{46}$ Girolamo CaraCAusi, Arabismi medievali di Sicilia, Palermo, 1983, p. 173, alla voce channacca. 
$\operatorname{araba}^{47}$ : tra i beni confiscati, un paio di orecchini con pietre e perle e parecchi anelli, due con ametista e smeraldo, due senza pietre, altri tre con pietre preziose.

Piccole sfere di pietre o metalli preziosi, in origine usati a scopo devozionale per le corone del rosario, i paternostri diventano ornamentali e lussuosi gioielli portati da uomini e donne. Di materiali svariati, dal corallo alla madreperla, dall'ambra alle perle, pendenti dalla cintura o attorcigliati alle borse, potevano servire da braccialetti o, pegni d'amore, usati come collane, talora intermezzati da grani più grossi, come nei rosari, in argento, oro, zaffiri, perle, balasci ${ }^{48}$ : Manfredi, oltre a un laqueum di seta verde con sette paternostri e a un paio de paternostris de seta violecta et filo de auro, a un partituri de paternostris in argento dorato in quadam gruppo, ne aveva di argento e corallo -quelli di corallo, "materia-colore" che simboleggia il sangue di Cristo, diffusi nell'isola soprattutto nel trapanese, patria della lavorazione ${ }^{49}$ - magnifico quello con novantasei perle, quattro zaffiri, tre balasci e quattordici bottoni d'argento ${ }^{50}$.

Alla cintura si appendevano anche piccole borsette di panno finissimo o velluto arricchite di pietre preziose, perle, argento, talora con delle scritte, come nella borsetta che pende dal fianco sinistro della dama vestita di broccato giallo, in basso a destra, nel Trionfo della Morte esposto nel quattrocentesco Palazzo Abatellis a Palermo ${ }^{51}$ : di velluto carmisino - il rosso di qualità più pregiata, dall'arabo qirmizî, "cremisi", derivato da qírmiz, cocciniglia $^{52}$ - era la burza confiscata a Manfredi, con un bottone di perle e quattro pietre.

Ricchissimo, l'inventario curato da Serra, di pietre preziose, una pecia con due zaffiri, un balaxio, un piccolo smeraldo e tre peciolas d'argento, e ancora undici pietre incastonate nell'oro: zaffiri, tre, e balasci (dall'arabo balahš), cinque grandi e tre piccoli, dal nome della provincia

\footnotetext{
${ }^{47}$ LANZADI SCALEA, Donne e gioielli, p. 99. Orecchini, anelli d'oro con pietre, verghe d'oro erano parte del tesoro rinvenuto da Margherita, liberta de partibus Romanie, Iris MIRAZITA, La truvatura di Margherita. Storia incompluta del ritrovamento di un tesoro (1341), in Trecento siciliano da Corleone a Palermo, Napoli, 2003, pp. 203 ss.

${ }^{48}$ Riccardo BEVERE, Vestimenti e gioielli in uso nelle province napoletane dal XII al XVI secolo, in ASPN XXII (1897), p. 320; LANZA DI SCALEA, Donne e gioielli, pp. $103 \mathrm{~s}$.

${ }^{49}$ Maria Concetta DI NATALE, Il corallo da mito a simbolo nelle espressioni pittoriche e decorative in Sicilia, in "L'arte del corallo in Sicilia", Palermo, 1986, p. 80. Sulla diffusione nella zona di Trapani, Fonti per la storia del corallo nel Medioevo mediterraneo, a c. di Aldo SPARTI, Palermo, 1986, $\mathrm{pp}$. $136 \mathrm{~s}$. Su qualità e utilizzazione del corallo trapanese, Genevieve et la mer", Tunis, 1985 , pp. 188 ss.

${ }^{50} \mathrm{Cfr}$. inventario in Appendice.

${ }^{51}$ Il "Trionfo della Morte" di Palermo. L'opera, le vicende conservative, il restauro, Palermo, 1989 , pp. 78 s., tavole 41 e 70 . Sul Trionfo della Morte di Palermo, immaginato "come un'enorme pagina miniata", Ferdinando BOLOGNA, Napoli e le rotte mediterranee della pittura da Alfonso il Magnanimo a Ferdinando il Cattolico, Napoli, 1977, pp. 11 ss. Sulla possibile identificazione del suo autore con Gaspare Pesaro, Geneviève BRESC-BAUTIER, Artistes, patriciens et confréries. Production et consommation de l'oeuvre d'art à Palerme et en Sicile occidentale (1348-1460), Roma, 1979, pp. 84 ss.

${ }^{52}$ CARACAUSI, Arabismi, pp. $158 \mathrm{~s}$. Sulle diverse qualità di rosso, Rosita LEVI PISETZKY, Storia del costume in Italia, II, Milano, 1964, pp. 148 ss.
} 
persiana del Balahšân, a indicare quella "sorta di pietra preziosa rossa, pallida, tirante al violetto, e che si suppone essere la matrice dei rubini" ${ }^{53}$.

Talora coperti da raffinati cappelli o da veli, più spesso raccolti in trecce, i capelli, elemento fondamentale della bellezza femminile, erano ornati con fili d'oro e perle a dare risalto incorniciandole alle fronti, alte e ampie secondo i canoni del tempo ${ }^{54}$. Parecchie nell'inventario le imperlatelle, lussuose acconciature di nastri e perle - un altro tipo erano le terzolle, composte da trecento perle ordinate in tre file - una con centosedici perle, una con quattro zaffiri, quattro balasci e centosette perle, ancora una con centoventicinque perle, sei zaffiri, quattro balasci, due custodite dentro un piccolo portagioie in legno, una cum seta nigra l'altra cum seta bioleta. Pezzi di raffinata oreficeria probabilmente siciliana ${ }^{55}$, l'imperlatella a dieci fili con dieci zaffiri e ottantaquattro perle e quella i cui fili di perle erano separati da piccole rose d'argento, cum partitorio cum rosetis de argento (l'inventario elenca un partitorium d'oro in dieci pezzi con pietre e perle, in totum completum).

Tra le acconciature e gli ornamenti da capo, comunissime le cajule, piccole cuffie utilizzate in Italia meridionale: catenelle o cordoncini che servivano ad adornare il capo secondo Salomone Marino, per Lanza di Scalea cajula sarebbe l'acconciatura chiamata dai francesi "coiffe" o quella specie di calotta che consisteva in un pezzo di stoffa, a volta tessuta in oro che racchiudeva la capigliatura, coperta da una reticella detta "crépine" 56 . In oro, seta, tutte di perle o con ornamenti di smalto, raccoglievano la capigliatura sulla nuca senza bisogno di essere legate sotto il mento perché fissate, ai capelli, da aghetti (in metalli preziosi e spesso anche questi ornati di smalti) ${ }^{57}$, sul capo, da un diadema arricchito di gemme o da un cerchio d'oro, il frontale, al quale venivano attaccate delle perle. Nell'inventario, una caxula de perlis cum duabus chirchellis, un'altra di perle cum cannolectis de auro $\mathrm{e}$ certos cannolectos de cayulam.

Sicilianizzazione probabile di infula le tre infurras de tela cilestra de capis: nei documenti trecenteschi il termine infula si trova talora utilizzato a

\footnotetext{
${ }^{53}$ CARACAUSI, Arabismi, p. 116. Sulle tecniche dell'oreficeria palermitana, Rita CEDRINI Tecniche tradizionali dell'oreficeria palermitana, in "Ori e argenti di Sicilia: dal'Quattrocento al Settecento", a cura di Maria Concetta DI NATALE, Milano, 1989, pp. 57-62. Sulla tecnica (decorazione, modellatura, cesellatura) dell'argento, Jole SCAVONE TRUPIA, L'argento e le sue tecniche, ivi, pp. 166-173.

${ }^{54}$ Dominique PAQUet, Storia della bellezza. Canoni, rituali, belletti, Trieste, 1997, pp. 36 ss.

${ }^{55}$ Sugli orefici siciliani, BRESC-BAUTIER, Artistes, pp. 113 ss. Caratterizzata, l'oreficeria siciliana medievale e poi moderna, dalla ricerca di effetti policromi raggiunti con due tecniche quella degli smalti sovrapposti ai fregi intagliati in lamina d'oro e quella delle gemme legate nei castoni intagliati nello spessore delle lamine d' oro e d'argento, Maria ACCASCINA, L ordinamento Istruzione", Milano-Roma, 1929.

${ }^{56}$ LANZA DI SCALEA, Donne e gioielli, pp. 106 s. Sulle pettinature femminili nel '300, LEVI PISETZKY, Storia del costume, II, pp. $123 \mathrm{sS}$.

${ }^{57}$ Levi PISETZKY, Storia del costume, II, p. 119. 
indicare le cuffie femminili, sia di tela bianca che riccamente ornate ${ }^{58}$. Con una derivazione probabile dal termine caracoles, erano vezzosi pettinini a forma di chiocciola i quattro caraculli de capillis inventariati.

Uso comunissimo, quello di corone e ghirlande d'oro e d'argento ma anche di cristallo e stagno, a testimonianza di un cambiamento che vedeva la corona diventare, da simbolo di potere e privilegio di classe, ornamento maschile e femminile diffuso indipendentemente dalla classe sociale ${ }^{59}$. Federico III nel novembre 1308 proibiva alle donne di portare gerlandam imperlatam et cum gemmis et auro che superasse la misura di due dita per lato, nelle ghirlande non potevano esservi merlature ${ }^{60}$. Veri e propri gioielli erano anche i merguli, staccati dal resto della corona ${ }^{61}$ (cui si potevano unire per mezzo di piccoli uncini) e che sulle corone si elevavano a somiglianza dei merli delle torri, sempre in numero dispari perché uno risultasse in centro, in mezzo alla fronte ${ }^{62}$ : nel marzo 1367, i messinesi Pietro Sardo e Matteo Regio avevano ricevuto, per un debito di 88 onze contratto da Federico IV per armare alcune galee, mergulum unum de corona nostra, munitum perlis et lapidibus preciosis ${ }^{63}$.

Il fratello di Manfredi, Artale Alagona aveva disposto del diadema regio, duas pecias corone regie che conservava ad opus et pro parte del re Federico $\mathrm{IV}^{64}$ : di corone d'oro - composte abbiamo visto da più parti unite per mezzo di uncinetti- e pezzi di corona, a Manfredi ne venivano confiscati in quantità, una devastatam consistentem in peciis undecim, con ventisette pietre preziose e ventiquattro perle inter magnas et parvas, una con undici merguli in cui erano incastonate centonove pietre preziose e centotre perle, una coronetam d'oro con undici merguli sontuosamente decorata con settantasei pietre e centotrentasei perle, una iorlandetam d'oro consistentem in dieci pezzi con un cordoncino in argento obliquo, in totum fornitam, un'altra con venti pietre e quaranta perle, un pecium de iorlanda d'oro con uno smeraldo, dodici pezzi di una corona d'oro con due perle e dodici pietre preziose, altri nove pezzi di corona con otto pietre e trentasei perle, un pezzo d'oro di una corona con nove perle e quattro pietre. Una ghirlanda d'oro con

${ }^{58}$ Levi PISETZKY, Storia del costume, II, p. 119. Sul significato di infula, a indicare genericamente le vesti sacerdotali ma anche, specificamente, certi ornamenti da capo, a mò di benda, DU CANGE, Glossarium, ad vocem.

${ }^{59}$ LANZA DI SCALEA, Donne e gioielli, pp. 108 ss.

${ }^{60}$ Testa, Capitula regni Siciliae, I, p. 91, cap. XCV.

${ }^{61}$ LANZA DI SCALEA, Donne e gioielli, pp. $110 \mathrm{~s}$.

${ }^{62}$ LEVI PISETZKY, Storia del costume, II, p. 131.

${ }^{63}$ ASP, $R$. Canc., reg. 10, c. 59 v. (13.3.1367). Tra gli oggetti dati in pegno in quegli anni da Federico'IV, una spadă ad modum Castille guarnita d'argento con il cinto adorno di perle ad arma nostra et Regis Aragonum, LANZA DI SCALEA, Donne e gioielli, p. 135. E ancora gioielli, saliere, biccheri, tazze, COSENTINO, Le nozze, pp. $27 \mathrm{~s}$.

${ }^{64}$ GIUFFrIDA, Il cartulario della famiglia Alagona, pp. 14, 54 s., doc. XXXIV. 
tredici fibbie, consistentem nuskis tresdecim, decorata riccamente con pietre, sessantatre, e perle, cinquantatre ${ }^{65}$.

Parecchie e di materiale vario piccole cassette, cofanetti che accompagnano la vita delle donne custodendo gioielli, ricami, fialette di profumo, gli oggetti più cari e i ricordi più intimi: due eburnee, una con un fregio decorativo in bronzo, l'altra listata in argento (in quest'ultima erano conservati sette anelli d'oro, quattro di pietre e tre di perle), una di legno inter quam est quoddam pomum de ambra munitum de argento cum quadam catinella, ancora una di legno che conservava due imperlatelle, una caxetam di legno guarnitam de argento cum laqueo de seta carmixina, cum aguglecta de argento e anulo de osso de perla. E ancora, una ad figuras con dentro due pettini d'avorio, un'altra ad figuras anche questa con due pettini eburnei e due bicchierini di cristallo. E una, estremamente delicata, di cristallo, a protezione della quale era un astuccio in legno. In un piccolo scrigno in legno dipinto musiatum erano conservati paternostri in argento e corallo.

E poi le buxule, una piccola in legno, le altre in vetro, avorio (una di quelle in avorio conteneva una dubla, moneta d'oro ${ }^{66}$ ), una in argento decorata con una piccola croce in superius: piccoli vasetti, bossoletti appunto in cui conservare unguenti profumati, medicine ma anche, come nell' inventario, le carte di famiglia più importanti e le reliquie ${ }^{67}$.

\section{VESTI E ACCESSORI}

Elegantissimo il ciprensem di velluto violato con ricami floreali in oro, cum arborectis de auro: la veste - forse sul tipo di quelle usate a Ciprospesso presente negli inventari siciliani, lunga sino ai piedi, dalle maniche lunghe e ampie e scollata da spalla a spalla, talora abbottonata davanti. Cipriane considerate indumenta inhonesta per il fatto che habent gulam tam magnam, quod ostendunt mammillas, et videtur quod dicte mammille velint exire de sinu earum ${ }^{68}$; a coprire la nudità che si lamentava ${ }^{69}$ spesso si poneva un mantello: nell'inventario sono elencati in un'unica voce ciprensem e mantello di velluto carmisino.

Veli di lino o seta, ad avvolgere testa, mento, collo scendendo sulla veste, spesso di seta tessuta a fili d'oro, con una grande varietà di forma $\mathrm{e}$ colori $^{70}$, erano le glimpe, tra i beni confiscati a Manfredi sedici glimpectas et mandilla riposti in una cassetta, un mandile di seta cum listis de oro e uno,

\footnotetext{
${ }^{65} \mathrm{Cfr}$. voce nusca in DU CANGE, Glossarium. Sulla fortuna delle perle nel ' 400 , favorita dalla preferenza per ornamenti delicati e luminosi, Maria Giuseppina MUZZARELLI, Gli inganni delle apparenze, Torino, 1996, p. 64

${ }^{66}$ Du CANGE, Glossarium, alla voce dubla e dupla n. 3.

${ }^{67} \mathrm{Cfr}$. inventario in Appendice.

${ }^{68}$ Citazione del Chronicum Placentinum (1388) da DU CANGE, Glossarium, ad vocem. Sulle cipriane, LEVY PISETZKY, Storia del costume, II, p. 97.

${ }^{69}$ LANZA DI SCALEA, Donne e gioielli, pp. $96 \mathrm{~s}$.

${ }^{70}$ Ibidem, pp.114 ss.
} 
sempre in seta, con fregi merlettati, cum frixis in capitibus $^{71}$ : incerto il significato del termine mandile, acconciatura di origine orientale, velo, o parte dell'abbigliamento (forse una specie di grembiule) ${ }^{72}$.

Sontuosi e dal regale ornato alcuni tra i mantelli di Manfredi: un mantitum firciatum di velluto e panno dorato, uno in seta murisca inbructato de auro, infoderatum di panno serico blevi (originario di Beauvais), uno di velluto rosso cremisino infiorato, inflorectatum, di perle, uno di seta bianca ricamato d'oro e foderato di zendado rosso. E ancora un mantum di seta infoderatum di panno di seta ialino e un bavarolum, risvolto di vestiti o mantelli da portare intorno al collo, di panno d'oro ${ }^{73}$.

Ornamento delle vesti doveva essere quella sorta di nodo in cui erano ammucchiate pietre e perle: in uno gruppo certos lapides et perlas, oltre a un gruppum con pezzi d'argento. Ornamentali anche i bottoni attaccati a maniche, cappelli, borse: uso smodato, al punto che Federico III nel novembre 1308 proibiva di portarne più di sette a vestito, senza superare il prezzo complessivo di $22 \operatorname{tar}^{\mathbf{}^{74}}$. Manfredi ne aveva grande quantità, in corallo, ambra, argento, perle, oltre a vari cappucci, uno di panno cilistrino con perle, uno di velluto verde infoderatum di panno di seta rosso, uno alla moda catalana, cathalaniscum, di panno dorato, due bunectos ${ }^{75}$ di panno rosso. Fino a un capellum de baptizari di seta ricamato, tarasiatum $^{76}$.

Parecchie le cinture: di seta violetta, de savasta, sabasto, ricercato e prezioso tessuto $^{77}$, di seta carmisina e oro, di seta chilestrina, di seta cilestra et biolata.

Inventariati dal camerario Serra anche vari gomitoli di filo prezioso: un glomarectum di seta, un glomarum $^{78}$ a filo argentato, un imboglum di filo argentato, due caxulas di filo et auro usitatas.

Il corpo del re era avvolto da stoffe e materiali che associano opulenza e varietà di colori: tra le cose necessarie a Martino il Vecchio per l'incoronazione due casse di filo d'oro, cento "caxes de fres", una "bandera de tercanell e d aur", dieci "penons de trempeta de tercanell groch e vermell ab lurs dorniments de flocadures e de cordons", "frens e selles que 1 dit senyor ha ops per la dita sua coronacio", sedici canne di tela "de rems primes e delicada"79.

\footnotetext{
${ }^{71} \mathrm{Cfr}$. inventario in Appendice.

${ }^{72}$ LanZa di SCAlEa, Donne e gioielli, pp. $113 \mathrm{~s}$. Cfr. voce mandile in Du CANGE, Glossarium.

${ }^{73}$ Sui lana, seta, pelli e i diversi materiali degli abiti, Françoise PIPPONIER-Perrine MANE, Se vêtir au Moyen Age, Paris, 1995, pp. 22 ss. In particolare sui diversi tipi di tessuti serici, LEVI PISETZKY, Storia del costume, II, p. 167.

${ }^{74}$ TeSta, Capitula regni Siciliae, I, p, 90, cap. XCI.

${ }^{75} \mathrm{Cfr}$. voce boneta in DU CANGE, Glossarium.

${ }^{76}$ Sull'abbigliamento infantile, LEVI PISETZKY, Storia del costume, II, pp. 191 ss.

${ }^{77}$ LANZA DI SCALEA, Donne e gioielli, p. 101.

${ }^{78}$ Du CANGE, Glossarium, ad vocem.

${ }^{79}$ Daniel GIRONA LlagosTERA, Itinerari del rey en Martì, (1396-1410), "Anuari de l'Institut d'Estudis Catalans" 1911-12, (4), p. 56 (4.1.1399). Su abiti e gioielli utilizzati da Ferdinando de Antequera in occasione della sua incoronazione, Roser SALICRU I LLUCH, La coronació de Ferran d'Antequera: l'organització i els preparatius de la festa, in "Anuario de Estudios Medievales", $25(1995)$, pp. 728 ss. 
Per Martino il Giovane, il camerarius acquistava dieci palmi di tela coloris $a z u l i{ }^{80}$, il sarto realizzava cappucci, scarpe, iupparellos di seta (zipparelli, tuniche maschili corte ai fianchi, simili ai farsetti), procurando la materia prima - pelli, velluto carmisino, panno de scarlato, de lira e di altri colori ${ }^{81}$ con una pezza de cammillocto nigro cuciva un manto de pluvia infurriato con inserti di panno verde fiorentino ${ }^{82}$. Colore nero che lungi dall'essere utilizzato per rendere visibile contrizione e tristezza, era impiegato perché così dettava la moda, accoppiato a vivaci e brillanti colori ${ }^{83}$.

Quando il miles Ximenes de Lerda aveva prestato alla Curia denaro pro accurrimento et subvencione solidorum gentis armigere servite all' assedio di Caccamo, aveva ricevuto in pegno un fermaglio rotondo in oro factum ad modum rote sancte Catherine con perle e pietre preziose ${ }^{84}$. Due nell'inventario i firmaglia d'argento e smaltati. Quasi sempre rotondi o lievemente concavi, con incastonate gemme di colore diverso, balasci, perle e smeraldi, i fermagli erano ornamento per le vesti, messi talora al centro della scollatura o appuntati su spalla sinistra e petto a chiudere il mantello ${ }^{85}$.

Mantelli di panno, cappe di velluto per cavalcare, oltre a giubbe e cotte di zendado o di ciambellotto, tuniche di panno di Firenze o di Damasco, formavano una cospicua parte dei corredi siciliani ${ }^{86}$. Mantelli non molto lunghi utilizzati per andare a cavallo, le cappe potevano essere di drappi costosi e ornate di fregi ricchissimi, foderate di seta o pelliccia o intessute in oro: tra quelle di Manfredi, una cappa de cavalcari di velluto chilestro stillato de auro infoderatam di panno serico rosso, una de diaspro foderata di panno serico rosso, altre due di seta chilestra imbructatis de auro ${ }^{87}$. Diventate oggetto di lusso, Federico III nel 1308 vietava alle donne di portare cappe pro equitando di sciamito, panno dorato o setato ${ }^{88}$.

${ }^{80}$ ASP, Misc. Arch., II, reg. 34, c. 108 r. (20.11.1398).

${ }^{81}$ ASP, Misc. Arch., II, reg. 34, c. 171 r. (1.5.1399).

${ }^{82}$ ASP, Misc. Arch., II, reg. 34, c. 77 v. (1.8.1398). Sulla preferenza dell'imperatore Federico per il colore verde, Salvatore Tramontana, Giochi, feste, spettacoli, in "Uomo e ambiente nel Mezzogiorno normanno-svevo" (Atti delle ottave giornate normanno-sveve, Bari, 20-23 ottobre 1987), Bari, 1989, p. 337.

${ }^{83}$ PIPONNIER; MANE, Se vêtir au Moyen Âge, p.144. Sul significato sociale dell'abito, Jacques LE GoFF, La civiltà dell'Occidente medievale, Torino, 1981, pp. $381 \mathrm{~s}$. Sui colori degl abiti e i loro significati, MUZZARELLI, Gli inganni, pp. 46 ss.

${ }^{84}$ ASP, Misc. Arch., II, reg. 34, c. 118 r. (31.1.1398).

${ }^{85}$ LANZA DI SCALEA, Donne e gioielli, p. 140; LEVI PISETZKY, Storia del costume, II, p 140. Sui due fermagli di Alfonso il Magnanimo (da un inventario del 1429) e sul significato di questo particolare gioiello, Margarita TINTÓ I SALA, Dos fermalls $i$ altres joies d'Alfons el questo particolare gioiello, Margarita TINTÓ I SALA, Dos fermalls $i$ altres joies d'Alfons el Magnanim, segons un inventari de la Taula de Canvi de la
Historica et Archaeologica Mediaevalia", 26, 2005, pp. $768 \mathrm{~s}$.

${ }^{86}$ Salvatore SALOMONE MARINO, Le pompe nuziali e il corredo delle donne siciliane, in ASS, n.s. I, fasc. I, Palermo, 1876, p. 218

${ }^{87} \mathrm{Cfr}$. inventario in Appendice.

${ }^{88}$ TeSTA, Capitula regni Siciliae, I, p. 91, cap. XCIII. Sulla disciplina suntụaria dal XIII al XV secolo, MUZZARELLI, Gli inganni, Torino 1996, pp. 99 ss. Sulle innovazioni trecentesche (maniche lunghe e strette, scarpe a punta, vesti aderenti e con strascico) a slanciare la figura enfatizzandone la verticalità, ivi, pp. 33 ss. 


\section{IL CORREDO}

Adibito, probabilmente, a conservare il corredo nuziale e la biancheria di qualità migliore - un paio di lenzuola de tela de arenzo, il tessuto di lino, candido e fine, detto "tela di rensa", dal nome della città d'origine, Reims -il caxonum ad arma de Aragona.

Uso tradizionale in Sicilia quello delle belle coltri, non solo di broccato ma ricamate con vari motivi: a onda o a pignola, a bottonelli o denarelli o pampine di arancio o a scacchi o a porta di Tripoli ${ }^{89}$. A Manfredi ne venivano confiscate una albam ad portam de Tripuli, una albam acumanu laboratam, fatta a mano con ago, una muriscam foderata di tela verde, una di panno dorato con una fodera di zendado rosso, una che poteva mettersi anche a muro: cultram unam seu paramurum de panno murisco di seta foderato di tela verde. Stesi sulle pareti a completare l'arredamento e ornare, un paramurum di panno dorato e velluto carmisino foderato con zendado verde, uno ferciatum d'oro e velluto rosso cum ferciis decem, un altro ferciatum d'oro e velluto rosso cum ferciis novem, uno consunto di zendado con fondo color oro, uno ficcatum d'oro e di velluto blavo cum ferciis septem foderato di tela verde.

Scarsa la biancheria personale che si limita a poche camicie, sul cui significato e diffusione non si hanno notizie precise ${ }^{90}$, quanto alla bellezza dipendeva dalla qualità della tela che poteva essere più o meno fine ${ }^{91}$ : raffinata una delle camicie di Manfredi, di tela lavorata con oro. Non semplice capire cosa fossero le tredici clunuolas orlate, imburdutas, di seta e oro, forse mutande femminili forate ${ }^{92}$.

Abbondante la biancheria da letto, varie paia di lenzuola di tela, alcune con inserti di seta e d'oro, cum listis de seta et de auro, un paio di seta alba cum listis de auro.

Per la cura e l'igiene personale, cinque asciugamani di cui quattro con una decorazione a rocchetto, roddichillata, i fazzoletti, dodici stuglabucha, parecchi pettini in materiali pregiati (talora intagliati con fregi, fungevano da oggetti ornamentali): tre d'avorio, uno in argento smaltato, altri due in avorio dentro una cassetta assieme a due cucchiaini di cristallo di cui uno rotto. E ancora, quattro scrimarolos in avorio, di cui tre piccoli e, a ravvivare la

\footnotetext{
${ }^{89}$ Enrico MAUCERI, Inventari inediti dei secoli XV e XVI, in ASSO, XXX (1915), pp. $105 \mathrm{~s}$.

${ }^{90}$ Tramontana, Vestirsi, pp. $135 \mathrm{~s}$.

${ }^{91}$ LeVI PISETZKY, Storia del costume, II, p. 144.

${ }^{92}$ Cluniculum è il foramen quod fit in camisiis feminarum circa inguina, vel generaliter quod fit in pannis earum circa latus, DU CANGE, Glossarium.
} 
pettinatura, un pettine de buxio, di bosso, legno duro e resistente dalla grana compatta e finissima ${ }^{93}$. Uno stuzzicadenti, dintiglerium, d'argento ${ }^{94}$.

A protezione e difesa, durante le torride estati isolane, da fastidiosi insetti, una zanzariera, musquectum, di tela.

Contribuivano ad arricchire di colori (i preferiti, come per l'abbigliamento, rosso, verde ma anche ialino, secondo la definizione di $\mathrm{Du}$ Cange, coloris vitrei) e tessuti le stanze del castello vari complementi d'arredo: nelle stanze destinate al riposo - lì si trovavano tre ricercati materassi di zendado rosso e ialino plena bombice - un supracelum ferciatum con velluto rosso e oro, una chilona, coperta in tessuto di Châlons, ialina, tre pezzi de cortina di zendado verde cum $^{\text {bucculis }}{ }^{95}$.

Parecchie le cortine: una di velluto rosso e panno dorato ferciatam, una di seta verde e rossa consistentbem in pecias quatuor, una di panno moresco, una lussuosa cortina e un imborlachium di panno rosso consistentem in quinque peciis. Una napam de toreo pictam, drappo, suppellettile da letto, dipinto $^{96}$.

Opulento e lussuoso simbolo della ricchezza degli Alagona, il copertorium de lecto de skinis de vayris ed un altro de armenis. Orpelli, rigonfiamenti, svolazzi negli abiti e non solo, sottolineati dalle fodere di pellicce, erano criterio di distinzione sociale ${ }^{97}$, in particolare la pelliccia di vaio -dal latino varius, "di colore variegato" - nome medievale dato al mantello di uno scoiattolo russo o siberiano d'inverno tutto grigio all'infuori del petto, bianco: la pelliccia che si presenta come una serie di scudi bianchi su fondo grigio ${ }^{98}$, era utilizzata per dare morbidezza a colli e maniche, foderare mantelli e profilare vesti, berretti, calzature ${ }^{99}$. Pregiate anche le pellicce di ermellino e zibellino, meno quelle di volpe e coniglio ${ }^{100}$, l'uso massiccio testimoniato dal fatto che - frivolezze mondane penetrate nei monasteri- il concilio di Londra del 1127 sancisse il divieto per le badesse di portare pellicce d'agnello e di gatto ${ }^{101}$. E se i Capitoli di Federico III di

\footnotetext{
${ }^{93}$ Sugli oggetti da toeletta e da bagno (dal nectaturo de denti in oro, all'annettaorecchi d'argento), Riccardo BEVERE, Arredi, suppellettili, utensili d'uso nelle province meridionali dal XII al XVI secolo, in ASPN, XXI, Napoli 1896, pp. $634 \mathrm{~s}$.

${ }^{94}$ Uno di corallo nell'inventario di beni del 1439, redatto a Palermo, di Caterina Abbatellis, Henri BRESC, Une maison de mots: inventaires palermitains en langue sicilienne (1430-1456), in "Bollettino del Centro di Studi filologici e linguistici sicliani", n. 18, Palermo, 1995, p. 142.

${ }^{95}$ Sulle parti che compongono il letto, BEVERE, Arredi, suppellettili, utensili, pp. 626-633 specificamente nelle case siciliane, BRESC, Une maison, p. 116. Su arredi e complementi d'arredo nelle case barcellonesi, Flocel SABATÉ, Els objectes de la vida quotidiana a les llars barcelonines al començament del segle XIV, in "Anuario de Estudios Medievales", 20 (1990), pp. 53 ss.

${ }^{96}$ Du CANGE, Glossarium, voce napa, drappo, e torale: "supellex lectaria, nam torum latini vocant, quodquid lecto instruendo ac insternendo conducit"

${ }^{97}$ Tramontana, Vestirsi, p. 81.

${ }^{98}$ Alessandro BARBERO- Chiara FrUgOnI, Dizionario del Medioevo, Roma-Bari, 2001, p. 251.

${ }^{99}$ Georges VIGARELlo, Lo sporco e il pulito. L'igiene del corpo dal Medioevo a oggi, Venezia, 1996, p. 63; MUZZARELLI, Guardaroba medievale, p. 361.

${ }^{100}$ MuZZARELLI, Gli inganni, p. 55. Sui tipi di pellicce di maggiore uso nel Mezzogiorno normanno-svevo, Annamaria NADA PATRONE, Pelli e pellami, in "Uomo e ambiente ne Mezzogiorno normanno-svevo", Atti delle ottave giornate normanno-sveve (Bari, 20-23 ottobre 1987), Bari, 1989, pp. 166 ss.

${ }^{101}$ Emanuele GALLO, Il valore sociale dell'abbigliamento, Torino, 1914, p. 159. ANUARIO De Estudios MEDIEVALES (AEM), 37/1, enero-junio 2007, pp. 71-106. ISSN 0066-5061
} 
Sicilia del 1308 avevano stabilito che solo dottori cuiuscumque professionis, giurisperiti, medici et non alii, potessero portare et induere vayros ad libitum eorum $^{102}$, alla fine del XV secolo l'uso delle pelli di vai presso la corte estense era tale che ne erano ornati gli abiti dei bambini e le coltri delle culle ${ }^{103}$.

\section{LA TAVOLA}

Colorate e raffinate le tovaglie de mensa: una di tela tarasiata con delle strisce cucite di seta carmisina e la parte inferiore decorata cum pedanis di seta carmisina, un'altra cum pedanis diversorum colorum, una ricamata con seta rossa e verde.

Non solo le tovaglie, anche i tovaglioli erano sovente impreziositi da ricami: un tovaglonum ad ramum, uno cum listis mayutis, altre due tovaglie da tavola cum listis mayutis ${ }^{104}$.

Parte fondamentale di una dote erano i servizi per la tavola: Eleonora di Sicilia, la terza moglie di Pietro IV, nel testamento destinava alla figlia gioielli e parte di un prezioso servizio d'argento per la tavola: una "barcha o pastera d'or, peça fastuosa del servey de taula, la vora de la quale peça estava guarnida ab quatre blasons de la Reyna y un del marit", mentre in una "peça d'argent en forma de castell se veyen figures d'home y de dòna en actitut de ballar". Barcha che serviva probablement "per a posar confits", anche se "s'hi posaven també ciris petitets, y, per altra part, curulla de roses", a ornamento appunto della tavola ${ }^{105}$. In occasione del Natale 1381, Sibilla de Fortià aveva regalato alla regina di Cipro vari pezzi di servizio da tavola, tra cui due grandi catini d'argento dorati e cesellati per lavare le mani, due grandi taglieri in argento, una coppa e un bicchiere d'argento dorato dentro e fuori, smaltati con i marchi della regina ${ }^{106}$.

Pezzi di un prestigioso servizio da tavola di Maria di Navarra e di Costanza d'Aragona dovevano essere la piccola saliera d'argento confiscata a Manfredi ad arma de Navarra, quella d'argento ad arma regalia, e una grande saliera in argento smaltata alla base, di Maria, ad arma Sicilie.

Un coperchio d'argento de picherio (l'inventario elenca coperchi di foggie varie: uno di seta virgulatum, un sopracoperchio di seta listata de auro), un grande tagliere d'argento su cui affettare le carni, una confettiera d'argento smaltata e dorata ad pedem era quanto restava di un elegante

\footnotetext{
${ }^{102}$ TeSTA, Capitula regni Siciliae, I, p. 89, cap. LXXXVIII.

${ }^{103}$ Carlo Merkel, Come vestivano gli uomini del "Decameron", Roma, 1898, p. 56.

${ }^{104} \mathrm{Cfr}$. inventario in Appendice.

${ }^{105}$ Ulla DeIBEL, La reyna Elionor de Sicilia, Barcelona, 1927, pp. 392, 416.

${ }^{106}$ LÓPEZ DE MENESES, Documentos culturales, pp. 740 s. Quarta moglie di Pietro IV il Cerimonioso dopo Maria di Navarra, Eleonora di Portogallo, Eleonora di Sicilia, già amante di Pietro, Sibilla de Fortià diventava sua moglie nel 1377: a Sibilla il re inviava una preziosa tavola di diaspro incastonata d'argento, ricca di perle, che poggiava su quattro piedi sostenuti da altrettanti leoncini scolpiti con finezza e, legati con catenelle, due bacili d'argento pesante che servivano per mescere acqua e vino, Alberto Boscolo, Bernardo Dez Coll, funzionario e cronisto

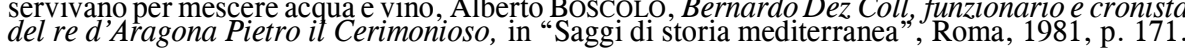


servizio per una tavola raffinata e regale a decorare la quale -assieme a tazze, bicchieri, piatti d'argento, scodelle, parecchi cucchiaini d'argento, d'argento dorato, quattro di cristallo (tutti rotti), una paletta in argento, un goctum de cristaldo ad pedem et cohoperchium in argento dorato - contribuivano due squisiti fiaschetti d'argento deauratos et ysmaltatos con il collo ornato da frange di seta verde e argento, cum chincis de seta virdi munitis de argento: vasi con un'ansa sola stretti e lunghi di collo, bordo ringrossato, larghi nel ventre, anche in vetro o terracotta, a conservare il vino ${ }^{107} \mathrm{o}$, probabile in questo caso, olio e aceto. di bronzo.

Forse una piccola fontana, o un recipiente per le mani, lo scurriachum

E ancora, coppe varie argentate e con coperchio, una deauratam cum coperchio smaltata alla base, vasi, recipienti, contenitori per sale o liquidi, boccali grandi e piccoli che fungevano talora da piccoli oggetti ornamentali: due coglarellis $^{108}$ di cristallo, una cannata de purchellana cum pede, coperchio e manico d'argento dorato e la cannatella d'argento smaltato e dorato ad arma Sicilie. Una piccola brocchetta di corallo, una maraxia de cristallo cum solo de argento, un'altra sempre di cristallo in una vesta ${ }^{109}$, uno starithum, fiaschetto forse, di cristallo con catenelle d'argento cum vagena, una caxecta de coreo ferrata in cui erano conservate due maraxie in argento dorate e smaltate ad arma regalia ${ }^{110}$.

Un marzapanum cum duobus purchillucis et cum filo de here et cum una buleta: la scatola, o più precisamente il "vaso fatto di legno sottile, per lo più di forma rotonda", ma anche in porcellana, in cui si poteva riporre qualsiasi cosa, dalle spezie alle confetture, derivante forse da Martaban, città birmana dove nel medioevo si esportava una celebre lacca' ${ }^{111}$. Questa di Manfredi aveva due conchiglie porcellanacee ai lati ${ }^{112}$, un filo in bronzo e una buleta, chiodo con capocchia larga e tondeggiante, di quelli non molto lunghi, che potevano essere di ferro, ottone, argento a seconda degli usi ${ }^{113}$.

\section{ARREDI E ORNAMENTI}

Dietro al gusto del Medioevo per i colori smaglianti "la paura della notte, la ricerca della luce che è salvezza"114: come quella delle vetrate rosse

\footnotetext{
${ }^{107} \mathrm{Cfr}$. voce flasca in Du CANGE, Glossarium.

${ }^{108}$ Cogla, pro cochlea era il vas quo bibitur, in formam cochlae confectum, Du CANGE, Glossarium.

${ }^{109}$ Definizione di cannata di Du CANGE, Glossarium, è cantharus, poculum, vas strictius et oblongius, Germanis et Belgis.

${ }^{110}$ Maracio era un tipo di vaso specifico per il sale, Du CANGE, Glossarium, dall'arabo marašša, "fiala", CARACAUSI, Arabismi, p. 280.

${ }^{111}$ CARACAUSI, Arabismi, p. 283.

${ }^{112} \mathrm{Cfr}$. voce porcelletto in Salvatore BATTAGLIA, Grande dizionario della lingua italiana, XIII, Torino, 1986

${ }^{113}$ Salvatore BATTAGLIA, Grande dizionario della lingua italiana, II, Torino, 1962, ad vocem.

${ }^{114}$ LE GoFF, La civiltà, p. 361.

ANUARIO DE ESTUDIOS MEDIEVALES (AEM), 37/1, enero-junio 2007, pp. 71-106. ISSN 0066-5061
} 
della cattedrale di Chartres dovuta probabilmente a un composto contenente dell'oro, la "porpora di Cassio"115. Applicato su diversi supporti, dal vetro alla ceramica al metallo, lo smalto - polvere cristallina vetrificabile al fuoco, ricavata dal vetro frantumato e pestato finemente, i colori ottenuti mediante ossidi di metalli che possono sia mantenere la trasparenza iniziale (smalti translucidi) che rendere opaco il fondente (smalti opachi)- può anche diventare colore per dipingere, steso con tocchi di differenti tonalità sulla superficie liscia del metallo. Fondamentale, la scelta del metallo, per gli smalti translucidi la cui brillantezza dipende in buona misura dalla luminosità della lamina di base, l'argento e l'oro per questo motivo i metalli più indicati. A dare luce e brillantezza, spesso sovrapposto a una lamina di metallo prezioso, lo smalto decora l'oro, l'argento, il rame, il bronzo, il ferro ${ }^{116}$. Vasta la gamma degli oggetti smaltati nell'inventario: si va dall'agnus Dei alle tazze, dal cultellucium con il manico d'argento smaltato al gotum d'argento, dalle spade con i manici d'argento smaltati alla coppa dorata con il coperchio smaltata alla base, dalle maraxie d'argento dorate e ysmaltate alla grande saliera smaltata ad pedem ad arma Sicilie de argento, inter quam sunt due carole d'argento smaltate (forse i coperchi della saliera, a forma di gruppi danzanti), dalla cannatellam d'argento smaltato e dorato ad arma Sicilie alla confiteriam d'argento smaltata, al mergulum di corona con sei smalti e ai due fermagli smaltati ${ }^{117}$.

Cultura, quella del Trecento, espressione di una società che aveva esaltato soprattutto "la sete di felicità terrena" ${ }^{118}$ : alla ricerca di un benessere che si nutre, anche, di piaceri tutti terreni, di begli oggetti che oltre all'espressione di uno status symbol, lusingano il tatto e la vista. Distribuiti a rendere più confortevole, caldo e al contempo lussuoso il castello degli Alagona, soprammobili di vario genere: la statua di un uomo selvatico che suonava il corno - quendam hominem silvestrem de argento deauratum, cum cornu in ore et quadam targa in pede in una vesta - il purchillucium de mari, conchiglia ornamentale, oviforme e con margini crenulati ${ }^{119}$, due stecchette, sicarelos, di avorio nero e bianco.

Molti cuscini, piccoli e grandi: otto coxinellos di panno dorato, tre di tela di cui due con bottoni in seta alba et violecta; uno di tela orlato con seta nera, mediam faciam di un piccolo cuscino torasito, ricamato, un coxinum ad arma de Aragona, uno di seta de panno murisco.

\footnotetext{
${ }^{115}$ Serge Hutin, La vita quotidiana degli alchimisti nel Medioevo, Milano, 1991, p. 179.

${ }^{116}$ Valérie GonzÁLEZ, Gli smalti dell'Europa musulmana e del Maghreb, Milano, 1994, pp. 17 s. Suii metodi di applicazione dello smalto e sugli smalti dipinti, ibidem, pp. 18 ss. Sull' equipaggiamento militare (dalle placche di bardature per cavalli, a staffe, speroni e armi) smaltato, ibidem, pp. 141 ss.

${ }^{117} \mathrm{Cfr}$. inventario in Appendice e la voce carola in Du CANGE, Glossarium. Sulla tradizione siciliana dei gioielli ornati con smalti, Maria Concetta DI NATALE Le vie dell'oro: dalla dispersione alla collezione, in "Ori e argenti di Sicilia: dal Quattrocento al Settecento", p. 24.

${ }^{118}$ Georges DuBY, L'arte e la società medievale, Roma-Bari, 1999, p. 302.

${ }^{119}$ Vincenzo MORTILLARO, Nuovo dizionario siciliano-italiano, r.a. Palermo 1969 dell'edizione del 1862, riporta la voce "purcidduzzi di mari", spiegandola come "sorta di testaceo, conche veneree". 
Parecchi gli oggetti di provenienza e fattura moresca, la cui diffusione nell'isola è testimoniata da una tavoletta del soffitto ligneo allo Steri di Palermo con due principesse che sorreggono un piatto moresco in cui al centro è collocato lo stemma dei Chiaromonte ${ }^{120}$. Manfredi Alagona aveva una coperta foderata con tela verde, parecchi cuscini, una coperta seu paramurum di panno murisco di seta foderato di tela verde, un mantello di seta murisca inbructato de auro, infoderatum de cindato blevi e un calamaio d'argento muriscum.

A illuminare le stanze, un candelabro d'argento fractum ad modum cope ad arma de Aragona. Ornamentali due piccoli alberi di corallo, diciassette di perle, uno d'oro, quello dell'albero motivo decorativo dalle molteplici simbologie - l'albero della vita, l'albero della scienza del bene e del male- che si ritrova nella cipriana di velluto viola cum arborectis de auro. E, soprammobili anche questi o forse candelabri, un arborectum con quattordici volute di serpente e altri due arbores di cristallo cum tornibus serpentis quatuordecim ${ }^{121}$.

Le armi: il tiniri de ense in avorio intaglatum, cum lenzo et pomo, vale a dire l'impugnatura della spada. Più decorative che di difesa le quattro spade con manici d'argento smaltati e punta d'argento (cum una bruccheta de argento $)^{122}$ e il piccolo coltello con manico d'argento dorato e smaltato, oggetto che si poteva portare legato alla cintura. Un vessillo, la bandera di zendado rosso fregiata con quattro sbarre d'argento: stemma simile a quello di una famiglia che come gli Alagona, si era trasferita dalla Catalogna alla Sicilia, i Villaragut ${ }^{123}$

Il gioco: varie tavole da gioco, taboleri, in materiali vari, dal legno di cipresso al fragile tabolerium di cristallo cum scaquis et tabolis. Oggetti di grandi dimensioni, lussuose e costose le scacchiere medievali -in legno pregiato, avorio, con decorazioni in oro, argento, ambra, diaspro, cristallo, perle- erano vere opere d'arte, i pezzi (rari quelli conservati) finemente decorati. Gioco degli scacchi che dall'area indo-persiana si diffondeva a seguito della conquista araba della Persia tra gli arabi e quindi in tutto l'Occidente europeo lungo due direttrici: attraverso l'Oriente bizantino verso Russia e Scandinavia, tramite la Spagna araba e probabilmente, la Sicilia,

\footnotetext{
${ }^{120}$ Ferdinando Bologna, Il soffitto della Sala Magna allo Steri di Palermo, Palermo, 1975, p. 89, tavoletta XIV a. Nel soffitto - "orgoglio" di una classe, quella nobiliare, affermatosi allo Steri fino al "narcisismo araldico" - lo stemma degli Allagona è inserito in un fregio di clipei e figure alate musicanti, ibidem, p. 169, tavoletta XXXII a., e p. 239.

${ }^{121}$ Sulla voce arbor nel significato di candelabro, DU CANGE, Glossarium e BEVERE, Arredi, suppellettili, utensili, p. 640. Sul significato di serpe come striscia luminosa o fiamma in movimento ("serpe di fuoco"), BATTAGLIA, Grande Dizionario, XVIII, Torino, 1996.

${ }^{122} \mathrm{Cfr}$. la voce brochia $\mathrm{n} .2$ in Du CANGE, Glossarium. Ferrante Domingo, subcamerarius di Martino il Giovane, faceva guarniri et armari per il re una lancia, pingi astam et forbiri necnon decurari ferrum, aggiungendo ornamenti in seta e bottoni in oro, ASP, Misc. Arch., II, reg. 35, c. 173 v. $(1.7 .1397)$.

${ }^{123}$ MANGo di Casalgerardo, Nobiliario di Sicilia, II, p. 252 s. e tav. LIII. 
nell'Occidente europeo ${ }^{124}$. Nel 1283, alla fine della sua vita, il re di Castiglia e León Alfonso X el Sabio (1252-1284), traduceva e riformava alcune regole arabe del gioco in un trattato dedicato agli scacchi, Libros del ajedrez, dados $y$ tablas, famoso codice miniato conservato all'Escorial ${ }^{125}$.

Grande il successo nella cultura cavalleresca, diffusissimi tra i nobili gli scacchi tradizionalmente, nell'iconografia come nella poesia, favorivano i colloqui d'amore, come in una tavoletta nel lato nord-est del soffitto dello Steri a Palermo raffigurante Tristano e Isotta che, sotto un padiglione sostenuto da pali, con un drappo damascato a motivi floreali e orlato di ermellino, giocano a scacchi ${ }^{126}$.

Scarno e di contorno parrebbe, dall'inventario dei beni di Manfredi, l'angolo per scrittura, studio, lettura: un calamaio d'argento cum pignarolo e pennam d'argento, sei libri e un signaculum d'argento con perle e pietre preziose. Una buxula d'avorio cum certis cartis scriptis.

\section{Altari e Celebrazione}

Probabilmente di fattura siciliana la caxecta de musia, a modo di mosaico, con inserti in argento e l'immagine della Vergine, la pecia in cui erano dipinti duo agnus Dei e la conecta de musia, piccola icona mosaicata, raffigurante san Giorgio, il martire cavaliere protettore di arcieri, cavalieri, militari rappresentato solitamente come un soldato in armi, spada e lancia in atto di sconfiggere un drago talora su un cavallo bianco con allusione alle sue irreprensibili doti di santo cavaliere: immagine raccolta con toni favolistici nella Legenda Aurea che fissatane la figura come quella di eroico cavaliere contribuiva a influenzare, oltre la fantasia popolare, l'ispirazione figurativa degli artisti, come nello splendido quadro di Paolo Uccello del 1455 conservato alla National Gallery di Londra, San Giorgio e il drago, con un'affilata principessa dal lungo strascico e le scarpe a punta. Patrono di Portogallo, Lituania, Inghilterra, Catalogna, gode di un culto speciale a Barcellona, splendida la statuetta d'argento di Sant Jordi in armi risalente ai primi del Quattrocento nella cappella della Diputació della città ${ }^{127}$.

Difensore delle armi degli Altavilla, nella cronaca del monaco Malaterra - che esaltando la gens normanna e le sue virtù, narra della

\footnotetext{
${ }^{124}$ Jean-Michel MEHL, Gioco, in Dizionario dell'Occidente medievale, a c. di Jacques LE GOFF-Jean Claude SCHMITT, Torino, 2003, I, pp. 478 ss. Sui giochi d'azzardo, in particolare dad e tavolette, messi al bando nell' 'isola da Federico III e su quenli permessi, TESTA, Capitula regn Siciliae, I, pp. 83 ss, capitoli LXXII-LXXXI. Cfr. Illuminato PERI, La Sicilia dopo il Vespro. Uomini, citta e campagne, 1282-1376, Roma-Bari, 1982, pp. 248 ss.

${ }^{125}$ Sul regno di Alfonso X, Salvador DE Moxó, Époça de Alfonso X, in Historia de España (fundada por) R. Menéndez Pidal, tomo XIII, La expansión peninsular y mediterránea (c 1212c.1350), vol I, La corona de Castilla, Madrid, $1990, \mathrm{pp}$. $91 \mathrm{ss}$. Sulla sua politica culturale, ibidem, pp. 183 ss.

${ }^{126}$ GABRICI- LEVI, Lo Steri di Palermo, pp. 116 s., 148 s.

${ }^{127}$ DE RIQUER, L'arnès del cavaller, tavole 194 e 195, pp. 216 e 217. Sull'interesse nutrito da Martino d'Aragona per le reliquie, in particolare quelle di san Giorgio, FODALE, Le reliquie, pp. $129 \mathrm{~s}$. 
conquista della Sicilia da parte dei Normanni di Ruggero d'Altavillasplendido cavaliere san Giorgio appare su un cavallo bianco a fianco del conte Ruggero nella battaglia di Cerami contro gli arabi, irrompendo nel punto in cui più fitti sono i nemici e con un vessillo bianco innalzato sulla lancia e sopra una croce luminosa, incita alla battaglia ${ }^{128}$. Patrono di Piana degli Albanesi, Modica, Sambuca di Sicilia, Prizzi, Vicari, san Giorgio è capillarmente presente nell'iconografia isolana: dall'affresco del palermitano Tommaso de Vigilia risalente alla seconda metà del Quattrocento conservato a Palazzo Abatellis che vede, sullo sfondo di una turrita città, il santo che libera la principessa, al dipinto San Giorgio che trafigge il drago custodito nella chiesa di Santa Maria della Catena di Termini Imerese in cui la firma dell'autore, Niccolò da Voltri, è apposta sul fodero della spada del cavalie$\mathrm{re}^{129}$. Protettore dei Chiaromonte che gli dedicavano una cappella, san Giorgio a cavallo riprodotto nel soffitto ligneo al piano superiore dello Steri e in un affresco al piano inferiore, affronta il drago per salvare la principessa ${ }^{130}$.

Tavolette di forma rettangolare con soggetti religiosi e ornamenti in avorio, rame, smalto venivano talora utilizzate come altarini ${ }^{131}$, tenute in camera da letto assieme a oggetti sacri ${ }^{132}$ come le reliquie: oltre a quelle in una conecta inclusas, l'inventario ne elenca custodite nella maniera più varia, dalla peciolam di panno di seta rosso alla buxulectam di vetro o alla piccola borsetta in cui erano racchiuse non meglio specificate reliquie sanctorum. A conferma di un'estrema varietà in cui accanto a reliquiari veri e propri - "gabbie trasparenti che permettono agli sguardi di arrivare fino ai corpi dei santi" consentendo, grazie a "quest'approccio visivo il rimedio alle proprie angosce" 133 - spesso arricchiti di gemme e pietre preziose, erano utilizzati i contenitori più vari: tondeggianti vasi di metallo lavorato a sbalzo e cesello, squadrati cofanetti di legno rivestiti di lastre d'avorio o d'argento dorato, ampolle in cristallo di rocca di fabbricazione fatimida, cassette bisantine in avorio e osso ${ }^{134}$.

Nel suo testamento Artale Alagona il Vecchio aveva disposto: volumus et mandamus quod in altaribus sive capellis in quibus habemus jus patronatus tam scilicet positis in ecclesia Sancte Agathe majoris quam veteris et alibi perseverent sacerdotes in celebratione divinorum cum perceptione soliti salarii

\footnotetext{
${ }^{128}$ Goffredo Malaterra, De rebus gestis Rogerii Calabriae et Siciliae comitis, ed. Ernesto PONTIERI, in Rerum Italicarum Scriptores, V/1, Bologna 1927, libro II, XXXIII, p. 44.

${ }^{129}$ Storia dell'Arte in Sicilia, I, Palermo, 1984, p. 176. Sulla leggenda, Iacopo da Varazze, Legenda aurea, I, Firenze, 2000, pp. 248 ss.

${ }^{130}$ Bologna, Il soffitto, p. 203; GABRICI-LeVI, Lo Steri di Palermo, p. 101.

${ }^{131}$ Maria ACCÁsCINA, Oreficeria Bizantina e Limosina in Sicilia, in "Bollettino d'arte del Ministero della Pubblica İstruzione", Milano-Roma, 1928, p. 552.

${ }^{132}$ Cfr. BEVERE, Arredi, suppellettili, utensili, p. 633.

${ }^{133}$ DuBY, L'arte e la società medievale, p. 294.

${ }^{134}$ Marco COLlaRETA, Arredi, suppellettili, decorazioni mobili, in Arti e storia nel Medioevo, II, Del costruire: tecniche, artisti, artigiani, committenti, Torino, 2003 pp. 309 s. Gemme che, viene ipotizzato, accrescevano il già miracoloso potere delle reliquie, Richard KIECKHEFER, $L a$ magia nel Medioevo, Roma-Bar1, 1993, p. 130. Sui "poteri" di pietre e gemme Adalberto PAZZINI, Le pietre preziose nella storia della medicina e nella leggenda, Roma, 1939. 
dati eisdem per nos annis singulis ${ }^{135}$ : profonda e sincera la devozione dei catanesi per sant'Agata - protettrice di balie, nutrici, fonditori di campane, tessitrici, invocata contro malattie del seno, incendi, eruzioni- le reliquie conservate in una cassa d'argento nella Cattedrale di Catania ${ }^{136}$, il velo oggetto di particolare devozione a difendersi dalla lava durante le eruzioni vulcaniche ${ }^{137}$. Portentose reliquie, velo e braccio di Agata erano state portate in processione dal vescovo catanese Simone del Pozzo a impetrare la vittoria per Artale Alagona: interrogato sull'ingresso a Catania di Artale Alagona e sulla condotta tenuta da Simone del Pozzo ${ }^{138}$, Filippo de Clerico Iohanne aveva riferito che durante quella processione il vescovo in vulgari eloquio aveva aveva detto: comu quistu velu ... liberau quista chitati de lu fochu a lu tempu de li pagani, acussi quistu velu possi liberari quista chitati de quistu periculu, imperò haiati quilla fidi qui appi li pagani a la liberacioni de lu fochu, acussì sancta Agatha liberarà quista chitati contra quillu qui la voli destruhiri ${ }^{139}$.

Legati gli Alagona, ai francescani: a Manfredi Alagona, il ministro dell'Ordine dei frati minori, frate Leonardo, nel 1376 dall'Aquila aveva inviato un saluto ringraziandolo per la devozione mostrata nei confronti dell'Ordine e invocando benedizione per lui, la moglie, i figli ${ }^{140}$. Nella storia di San Placido scritta dal secondo abate Marino de Guercis diretta a papa Bonifacio IX, in cui venivano fornite informazioni sulle condizioni dei monaci del monastero e la loro vita, si dice a un certo punto di monaci e sacerdoti che Artale e Manfredi Alagona avevano trasferito, transduxerunt, da quel monastero ad certa eorum monasteria dello stesso Ordine edificati per eos in eorum locis ${ }^{141}$.

Le spese per la cappella di palazzo in genere erano consistenti: tra gli arredi della cappella di Eleonora di Sicilia a Barcellona, due piccoli altari d'argento portatili, uno con le immagini smaltate di Maria e san Giovanni, l'altro "una imatge de la Mare de Déu y les armes d'Elionor"142. Nel 1348 il tesoriere di Eleonora, Ramón Savall, aveva pagato ad un argentiere valenzano, Pere Bernes, il valore di una naveta de argent, tota plana con due smalti

${ }^{135}$ LA LuMIA, Estratti,.pp. 187 ss.: Artale istituiva fidecommissari l'abate dị S. Nicolò de Arena e del monastero di Santa Maria de Nova Luce, Manfredi Alagona, il protonotaro Bartolomeo de Incorio.

${ }^{136}$ Alfredo CatTABiani, Santi d'Italia, Milano, 1993, p. 28.

${ }^{137}$ Patrizia SARDINA, Tra l'Etna e il mare. Vita cittadina e mondo rurale a Catania dal Vespro ai Martini (1282/1410), Messina, 1995, pp. 308 ss.

${ }^{138}$ Il 10 luglio 1392 Martino aveva ordinato al giudice della magna curia e luogotenente del giustiziere Tommaso Crispo di svolgere un'inchiesta - mala que fecit, predicavit et dixit - sulla condotta tenuta dal vescovo catanese in occasione dell'ingresso in città di Artale il 24 giugno 1392: sull'inchiesta, Fodale Scisma, pp. 53 ss. I vari passaggi del processo sono trascritti da R STARRABBA, Processo di fellonia contro Frate Simone del Pozzo, vescovo di Catania, in ASS, I (1873), pp. 174-200, 399-422.

${ }^{139}$ ASP, Protonot., reg. 4, cc. 164-165 r. (13.7.1392).

${ }^{140}$ GiUfFRIDA, Il cartulario della famiglia Alagona, pp. 88 s. (12.6.1376).

${ }^{141}$ ASP, Tabulario di S. Maria Maddalena, perg. 646 (27.5.1394).

${ }^{142}$ DEIBEL, La reyna Elionor, p. 423. Scudo bipartito quello di Eleonora, catalano-aragonese sul lato sinistro, di Aragona-Sicilia, con le aquile, sul destro, Nobiliario de la Corona de Aragón, I, p. 144 . 
nella copertura e gli stemmi del re Pietro e della regina Eleonora, e di un cucchiaino d'argento che servivano per la cappella di Eleonora. Tre anni più tardi, Pietro IV ordinava al baiulo generale di Catalogna di pagare quanto speso per tre inginocchiatoi per la cappella di palazzo di Barcellona, due dei quali ordinati per gli altari dedicati a san Bartolomeo e a san Nicola ${ }^{143}$. Nel 1376 il re chiedeva all'abate di Santes Creus due colonne di pietra vermella che si trovavano nella chiesa del monastero e una lapide della stessa pietra che dovevano servire, ancora, per la cappella reale di Barcellona ${ }^{144}$.

Probabile che assieme a tutto il resto, alcuni degli arredi di cappella delle regine di Sicilia, di Maria soprattutto, fossero confluiti tra i beni degli Alagona che comunque dovevano avere una cappella privata. L'inventario comprende una fetta cospicua di arredi sacri: un calice d'argento, due ampolline d'argento, una campanella e un incensiere, entrambi d'argento, una navetam d'argento ${ }^{145}$, un'immagine in alabastro della Vergine: utilizzato in lastre sottili per le vetrate, l'alabastro con la sua capacità di riflettere la luce rendendola di diafana bianchezza, era il materiale più adatto alla raffigurazione della Vergine Maria "luce del mattino" e, appellativo conferitole nel medioevo, "stella mattutina".

Lussuoso e completo il guardaroba liturgico compreso tra i beni confiscati, guardaroba per il quale erano solitamente impiegati materiali pregiati, soprattutto tessuti dorati e sete, tanto da superare talora in lusso le vesti principesche $^{146}$. Non solo l'abbigliamento pontificale ricco di stoffe seriche, dorate, di ornamenti e ricami a motivi geometrici e talora di carattere profano (per esempio animali esotici) ma anche quello di vescovi e alti prelati, vigente tra i chierici la tendenza a adottare, confondendosi con i laici, vesti ricercate e vistose: emblematici in tal senso i provvedimenti emanati nel Trecento volti a disciplinare l'abbigliamento degli ecclesiastici ${ }^{147}$.

L'inventario comprende un admictum -il primo dei sei indumenti comuni a vescovo e presbiteri (amictus, alba, cingulum, stola, manipulus et planeta $)^{148}$, indossato sotto l'alba che, di forma quadrata o rettangolare, circondava il collo e formava una sorta di colletto ${ }^{149}$, un'alba de presbitero, il bianco camice liturgico di colore bianco, una casubula di seta: la casula, veste sacerdotale posta su tutte le altre vesti, a forma di mantello chiuso ai lati e con una sola apertura alla sommità per introdurvi il capo, detta anche pianeta quia instar parve casae totum tegit, et signat caritatem $^{150}$. E ancora, due stole, le strisce di stoffa che giravano intorno al collo ricadendo sul petto,

\footnotetext{
${ }^{143}$ LÓPEZ DE MENESES, Documentos culturales, pp. 685 s., 687 s.

${ }^{144}$ Ibidem, p. 720.

${ }^{145}$ Cfr. . Du CANGE, Glossarium, ad vocem, n. 2, sull'uso del termine navis nel senso di navicula, "in qua thus asservatur".

${ }^{146}$ PIPONNIER-Mane, Se vêtir au Moyen Âge, p. 141.

${ }^{147}$ LeVI PISETZKY, Storia del costume, II, 201 ss.

${ }^{148} \mathrm{Cfr}$. voce amictus in Du CANGE, Glossarium.

${ }^{149}$ PIPONNIER-Mane, Se vêtir au Moyen Âge, p. 141.

${ }^{150}$ Du CANGe, Glossarium, ad vocem, n. 2. 
spesso finemente ricamate - con quell'arte dell'ago detta "opus anglicanum"- con figure di santi ${ }^{151}$ e, piccole salviette di stoffa che il sacerdote teneva durante la funzione sul braccio sinistro, due manipule, ad asciugare il sudore e usare come fazzoletto ${ }^{152}$

Destinata alle celebrazioni che si svolgevano all'esterno, una cappa de presbitero di panno dorato, foderata di zendado rosso: l'ampia veste liturgica di stoffa pregiata a forma di grande mantello, chiamata anche piviale, aperta sul davanti e fermata al petto da un fermaglio, dotata di un cappuccio rimpiazzato alla fine del medioevo da una fodera di panno pregiato o ricamato. Quanto ai colori, tendeva a prevalere il rosso, simbolo del sangue di Cristo e di quello dei martiri, unito al bianco, il colore della luce e del divino ${ }^{153}$.

Altrettanto sfarzoso il corredo per rivestire l'altare: un palium de diaspro foderato di tela verde, un pannum de altari de diaspro imbrustatum de auro, tre tovaglie de altari, un frontale de altari di seta (il velo per ornare la fronte dell'altare), un pallium de altari di seta infoderatum de tela chilestra, un pannum de camuca ${ }^{154}$ de altari foderato di tela chilestra.

Conservati in un astuccio di velluto, investam unam de billuto cum corporalibus, i corporali: quadrati di lino stesi al centro della mensa dell'altare per poggiarvi gli arredi sacri, come il calice d'argento deauratum cum pathena confiscato a Manfredi. Recipiente per l'acqua benedetta il sichium d'argento ${ }^{155}$.

E ancora, un libricellum di preghiere, otto libri ecclesiasticos ${ }^{156}$, un rollum de ere in quo sunt scripte hore sancte Marie: rotolo liturgico in cui erano state incise sul bronzo le preghiere per la Vergine ${ }^{157}$.

Al momento della consegna al camerario Guglielmo Serra del tesoro inventariato, dai beni in elenco erano spariti, casualiter amissa, una piccolissima cassettina rivestita d'avorio con inserti in argento dentro cui erano riposte un paio di lenzuola di lino e una pecia cortine di panno vermiglio. Non reclamate: eadem vobis relaxamus et remictimus graciose et nullam volumus vobis exinde fieri questione.

Data di recezione dell'articolo: aprile 2006

Data di accettazione e versione finale: decembre 2006

\footnotetext{
${ }^{151}$ LeVi PisetZKY, Storia del costume, II, 203.

${ }^{152} \mathrm{Cfr}$. voce mappula in Du CANGE, Glossarium.

${ }^{153}$ PIPONNIER-MANE, Se vêtir au Moyen Âge, pp. 140, 144.

${ }^{154} \mathrm{Dal}$ persiano kämhâ, la voce camuca arrivava al siciliano attraverso l'arabo kamhâa "vestis scutulata Damascena", tessuto in seta damascata importato dall'Oriente, CARACAUSI, Arabismi, p. 152 .

${ }^{155} \mathrm{Da}$ sicula, tipo di vaso ad usum ecclesie, Du CANGE, Glossarium.

${ }^{156} \mathrm{Cfr}$. inventario in Appendice.

${ }^{157}$ Sui significati di rotulus, o rollus, DU CANGE, Glossarium, ad vocem.
} 


\begin{abstract}
APPENDICE
L'inventario è stato rinvenuto presso uno dei registri del fondo Real Cancelleria dell'Archivio di Stato di Palermo: il registro numero 18 che consta di 140 carte (159 in base alla nuova numerazione) che misurano 29 centimetri di lunghezza e 21 di larghezza, pervenuteci nel complesso in buone condizioni. Il primo documento del registro è del 4 marzo 1393, l'ultimo del 25 gennaio 1394, anni focali per l'avvio di nuovi equilibri seguito all'arrivo dei Martini nell'isola.

Fatta la trascrizione, ci si è imbattuti in un nota di Lanza di Scalea ${ }^{158}$ in cui, parlando di cura e pulizia del corpo, si fa riferimento a un pettine d'argento smaltato "ricordato da un inventario signorile del secolo XIV": il rimando è a una "nota" di Giuseppe Silvestri — "Sullo stato e sulla riforma della legislazione dei pubblici Archivi: Apocha Guillelmi Catrilla (1392), p. LXXXIII, nota (1)"- senza l'indicazione di luogo e anno di pubblicazione, o ulteriori indicazioni. Dopo vari tentativi, si è riusciti a individuare il testo di Silvestri citato da Lanza di Scalea. Si tratta di uno scritto del 1870 in cui l'autore parlando degli archivi italiani e dell'idea di un lessico da "formare" "sopra i documenti nazionali", si sofferma su alcuni inventari di "suppellettili", pubblici o privati, che ricorrono spesso nei registri notarili e in quelli degli uffici pubblici palermitani. Tra questi ultimi, continua Silvestri, una "serie specialissima" sono gli inventari compilati "nell'interesse del fisco, in cui vanno descritti i beni mobili rinvenuti nelle magioni de' feudatari messi al bando per fellonia o per talento partigiano", inventari che, scrive Silvestri, dovrebbero trovarsi principalmente nei registri della Conservatoria del Real Patrimonio o della Secrezia di Palermo, ma anche nella Real Cancelleria. E qui, come esempio degli inventari di beni confiscati "di quelli de' quali avesse direttamente disposto la sovrana potestà", in nota Silvestri inserisce un inventario in cui si descrivono " $\mathrm{i}$ ricchissimi arredi confiscati nella sfarzosa magione del ribelle" Manfredi Alagona, commentando: "non so comprendere come possa essere sfuggito alla nota sedulità del Gregorio"159.

La lingua dell'inventario è un latino volgarizzato, con molte tracce di termini siciliani (ad esempio l'uso del termine scrimalem contemporaneamente a quello di pectinem; conam, arrivata al siciliano dal greco), e numerose varianti (cuxinellos, coxinellos; ysmaltato/ismaltato; caxectam/caxetam; biridi/virdi, viridi; cincthum/cinthum; zafiro/zefiro).

La scrittura è una gotica corsiva chiara e ordinata, la filigrana rappresenta la lettera "R", bombata e attraversata in centro da una linea retta, come quella indicata da Briquet al numero 8924 (citati gli esempi di Arezzo nel 1358, Venezia e Treviso nel 1359), e per la quale viene citata come "variante" quella di "Palermo, 1392"160.

\footnotetext{
${ }^{158}$ LANZA DI SCALEA, Donne e gioielli, p. 274, nota 300.

${ }^{159}$ Giuseppe SILVESTRI, Sullo stato e sulla riforma della legislazione dei pubblici archivi in Italia, Palermo, 1870, pp. LXXXIII-XCIV, nota 1: pubblicato pure in Rivista Sicula di Scienze, Letteratura ed Arti, a. IV, vol. VIII, Palermo 1872, pp. 237-268.

${ }^{160}$ BRIQUET, Les Filigranes. Dictionnaire historique des marques du papier, Paris 1907, t. III, p. 477 .
}

ANUARIO DE Estudios MedieVALES (AEM), 37/1, enero-junio 2007, pp. 71-106. ISSN 0066-5061
\end{abstract}


1393 febbraio 28, I indizione, Catania

Martino e Maria, re e regina di Sicilia, Martino, duca di Montblanc, rilasciano a Guglielmo Ça Trilla, mercante barcellonese, ricevuta per $i$ beni appartenuti a Manfredi Alagona consegnati al camerario della regina Guglielmo Serra, beni di cui lo stesso Serra ha stilato un inventario.

[ASP, $R$. Canc., reg. 18, cc. 18-21]

Apocha Guillelmi Çatrilla.

Pateat universis quod nos Martinus et Maria etc. et infans Martinus etc. confitemur et recognoscimus vobis, fideli nostro Guillelmo Çatrilla mercatori barchinonensi quod habuimus a vobis qui de nostro acceperatis mandato de bonis que fuerunt Manfridi de Alagona queque iussu nostri tradidistis fideli camerario nostro dicte regine Guillelmo Serra, prout fuimus per eum plenissime informati et constat per inventaria facta per eundem, res et bona sequencia, videlicet:

copam unam deauratam cum coperchio cum ysmaltis ad pedem. pedem.

Item gotum unum de argento deauratum cum coperchio cum ysmaltis ad

Item aliam copam de argento ysmaltatam cum coperchio ad pedem.

Item aliam copam de argento ysmaltatam cum coperchio ad pedem.

tam.

Item aliam copam de argento deauratam ad pedem cum coperchio ysmalta-

Item flasconos duos de argento deauratos et ysmaltatos cum chincis de seta viridi munitis de argento.

Item saleriam unam de argento parvam ad arma [c. 18 v.] de Navarra.

Item buctones de argento decem cum petris clavatos.

Item pomum unum de cristaldi de ense.

Item coperchium unum de argento de picherio.

Item coronam unam de auro devastatam, consistentem in peciis undecim cum lapidibus preciosis XXVII et perlis XXIIII ${ }^{\text {or }}$, inter magnas et parvas. lapidibus.

Item peciolum unum de auro de corona cum novem perlis et quatuor

Item anulos duos cum lapidibus preciosis, scilicet amastita ${ }^{161}$ et ysmeralde. Item caxitam unam de ebore.

Item caxulam unam de perlis cum duabus chirchellis.

Item iorlandam unam de auro consistentem nuskis tresdecim in quibus sunt lapides LXIII et perle LIII.

Item coronam unam de auro cum mergulis undecim in quibus sunt lapides CVIIII et perle CIII.

Item coronetam unam de auro cum mergulis undecim in quibus sunt lapides LXXVI et perle CXXXVI.

Item partitorium unum de auro consistens in decem peciis cum lapidibus et perlis, in totum completum.

Item iorlandetam unam de auro consistentem in peciis decem cum laqueo de argento obliquo, in totum fornitam.

\footnotetext{
${ }^{161} \mathrm{Per}$ amathista. 
Item aliam coronam de auro cum mergulis novem in quibus sunt lapides ${ }^{162}$ LXXVIIII et perle CIII.

Item iorlandetam unam de auro cum lapidibus XX et perlis XXXX.

Item caxonum unum ad arma de Aragona.

Item salariam ${ }^{163}$ unam magnam ysmaltatam ad pedem ad arma Sicilie de argento, inter quam sunt due carole de argento ysmaltate.

Item maraxiam unam de cristallo cum solo de argento. deaurato

Item cannata una de purchillana cum pede, coperchio et manico de argento deaurato.

Item copam unam de perle cum pede de argento et munitam de argento

Item cannatellam unam de argento ysmaltato et deaurato ad arma Sicilie.

Item copam unam de mazaro cum pede de argento deaurato.

Item confiteriam unam de argento ismaltatam et deauratam ad pedem.

Item tiniri unum de ense de ebore intaglatum, cum lenzo et pomo.

Item caxetam unam de ebore cum barris de ere.

Item caxetam unam de ligno guarnitam de argento cum laqueo de seta carmixina, cum aguglecta de argento, cum anulo de osso de perla, inter quam est quedam caxula incepta de seta.

Item peciolam unam de cindato rubeo, inter quam sunt reliquie sanctorum.

Item burxiam unam de carmixino veterem et quoddam trocium sete albe.

Item buxulam unam de ligno inter quam sunt tres carole de balcimo ${ }^{164}$ et duobus lapidibus pregnis.

Item conectam unam parvam.

Item caxectam unam de ebore barriatam de argento, inter quam sunt anuli septem de auro, quatuor de petris et tres de perlis.

Item tabolerium unum de cipresso.

Item coxinellos octo de panno deaurato.

Item cuxinellos duos de tela.

Item pecia tria de cortina de cindato viridi ialiano ${ }^{165}$ cum bucculis.

Item capuchium unum de billuto biridi infoderatum de cindato rubeo.

Item ciprensem unum de seta cum mantico de seta morisca.

Item ciprensem unum longum de panno de auro.

Item mantum unum de auro.

Item par [c. 19 r.] unum lintiaminum alborum de tela.

Item par aliud lintiaminum de tela de arenzo.

Item par aliud lintiaminum alborum cum listis de seta et de auro.

Item paramurum unum ficatum usitatum de cindato cum fundo de auro.

Item alcandoria duo de seta cum barris inter quandam caxicam.

Item cuxinos duos de seta et billuto ad arma de Navarra.

Item paramurum unum ficcatum de auro et de billuto blavo cum ferciis septem infoderatum de tela viridi.

Item par unum linthiarum de seta alba cum listis de auro.

Item paramurum unum de panno de auro et billuto carmixino cum ferciis decem septem ad arma de Navarra infoderatum de cindato viridi.

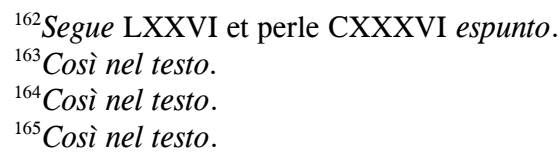


Item cuxinum unum de seta de panno murisco.

Item musquectum unum de tela.

Item par unum de cuxinellis de tela cum buctonis de seta alba et bioleta.

Item cuxinellum unum de tela tarasiatum de tela de auro.

Item cuxinellum unum de tela alba.

Item mesale ${ }^{166}$ unum et tuvaglonum unum ad ramum.

Item mensale cum listis mayutis.

Item mensale aliud usitatum.

Item manticum unum de billuto carmixino inflorectatum de perlis.

Item cultram unam albam ad portam de Tripuli.

Item cultram aliam albam acumanu laboratam.

Item gotum unum de argento deauratum.

Item caxetam unam de figuras.

Item buxuletam unam de vitro cum certis reliquiis.

Item cinchtum unum de seta bioleta.

Item cinchtum unum de savasta.

Item aliud pecium de cincto.

Item peciolum unum de seta et de auro.

Item pectinem unum de argento ismaltatum.

Item quatuor caraculli de capillis.

Item sex alii carraculli ${ }^{167}$ de capillis.

Item cincthum unum de seta carmixina et auro.

Item aliud cinthum de seta chilestrina.

Item glomarectum unum de seta.

Item agnus Dei de argento deaurato cum laqueo de seta carmixina.

Item alius agnus Dei ysmaltato cum una cruceta de argento.

Item cultellucium unum cum manico de argento deaurato et ysmaltato.

Item par unum de paternostris de seta bioleta et filo de auro.

Item par unum de paternostris de erba.

Item libricellum unum de oracionibus.

Item napam unam de toreo pictam.

Item purchillucium unum de mari.

Item rollum unum de ere, in quo sunt scripte hore sancte Marie.

Item buctonos novem de ambra.

Item pedem unum cristaldo munitum de argento.

lapidibus

Item burzam unam de billuto carmixino cum uno buctono de perlis et quatuor

Item capellum unum de baptizari de seta tarasiatum.

Item gotum unum de argento ysmaltatum et deauratum.

Item buctonos novem de perlis.

Item arborectos de perlis decem et septem.

Item glomarum unum de filo de argento. de argento.

Item gladios quatuor cum manicis de argento ysmaltatis, cum una bruccheta

Item buxulectam unam de ligno.

Item caxectam unam de ligno inter quam est quoddam pomum de ambra munitum de argento cum quadam catinella.

\footnotetext{
${ }^{166}$ Così nel testo.

${ }^{167}$ Cosi nel testo. 
Item caxectam aliam de ligno inter quam sunt due imperlatelle, una cum seta nigra et reliqua cum seta bioleta.

Item [c. $19 \mathrm{v}$.] imperlatellam aliam cum seta bioleta cum partitorio.

Item buctonos de perlis parvos inter quandam peciolam LIIII.

Item buxulam unam de ebore cum uno buctono de ambra munito de argento. Item conectam unam de musia cum ymagine sancti Georgi.

Item scrinetum unum de ligno pictum musiatum in quo est quoddam par de paternostris de argento et curallis.

Item imboglum unum de filo de argento.

Item calamarium unum de argento cum pignarolo et pennam de argento.

Item sicarelos duos de ebore nigro et albo.

Item caxulas duas de filo et auro usitatas.

Item buxulam unam de ligno.

Item cuxinos tres de seta murisca.

Item par unum linthiolum alborum.

Item tovaglas duas de mensa cum listis mayutis.

Item tovaglonum unum cum listis mayutis.

Item camisiam unam de tela ${ }^{168}$ laboratam de auro.

Item clunuolas imburdutas de seta et de auro XIII.

Item capucheum unum de panno cilistrino imperlatum.

Item banderam unam de cindato rubeo cum barris quatuor de argento.

Item cuxinellum unum de tela cum seta nigra urlatum.

Item tobaliam unam de tela tarasiatam.

Item aliam tobaliam de tela tarasiatam cum pedanis de seta carmixina.

Item aliam tobaliam de seta tarasiatam cum pedanis diversorum colorum. Item copam unam de argento deauratam cum coperchio ysmaltato.

medio.

Item taciam unam de argento intus dirutatam longam cum uno ysmalto in

arma de Aragona.

Item candelabrum unum de argento deauratum, fractum ad modum cope, ad

Item navem unam de argento deauratam cum carrochiis.

Item arborectum unum de auro.

Item picherium unum de argento deauratum vetus.

Item tacias deauratas de argento, duas cum ysmaltis et reliqua non.

Item platellos duos de argento.

Item taglerium unum de argento magnum.

Item scutellas duas de argento magnas.

Item starithum unum de cristaldo cum catinellis de argento cum vagena.

Item copam unam de perla munitam de argento.

Item saleriam unam de argento ad arma regalia.

Item goctum unum de cristaldo fractum. deauratum.

Item alium goctum de cristaldo ad pedem et cohoperchium de argento

Item coclarellas $\mathrm{IIII}^{\text {or }}$ de argento, quarum esta una deaurata.

Item palectam unam de argento.

Item $^{169}$ arborectum unum de cratallo ${ }^{170}$ cum tornibus serpentis quatuordecim.

\footnotetext{
${ }^{168}$ Segue tarasiatam espunto.

${ }^{169}$ Segue bo espunto.

${ }^{170}$ Per cristaldo.
} 
Item glimpectas et mandilla sexdecim in una caxecta.

Item pectinem unum de buxo.

Item stuglabucca duodecim.

Item facitergium unum.

Item peciolum unum de tela tarasiatum.

Item mediam faciam de cuxinello torasito.

Item scrimale unum de ebore.

Item pecium unum de here ad arma Aragonum.

Item tabolerium unum.

Item scurriachum unum de here.

Item aliud scurriacum de here.

Item conam unam ad pecias duas.

Item tabolerium unum de cristaldo cum scaquis et tabolis.

Item tabolerium aliud de cristallo cum scaquis et tabolis.

Item caxectam unam de coreo ferratam, in qua sunt due maraxie de argento deaurate et ysmaltate ad arma regalia.

Item chintum unum de sabastra.

Item buxulectam unam de mazaro munitam de argento.

Item mandille ${ }^{171}$ unum de seta cum listis de oro.

Item tobaliam unam tarasiatam cum pedacis ${ }^{172}$ de seta.

Item aliud mandile de seta cum frixis in capitibus.

Item caxectam unam ad figuras cum duobus pectinibus de ebore et duabus

coglarellis de cristaldo, una est fracta.

[c. 20 r.] Item buxulam unam de ebore cum una dubla.

Item cinctam unam.

Item cinchtum unum de seta cilestra et biolata.

Item bipariam unam imburdutam in quadam pecia.

Item burciam unam de panno de auro.

Item caxectam unam de coreo, inter quam sunt mandila sex.

Item pecium unum de palio de panno aureo.

Item firmaglia duo de argento et almatata ${ }^{173}$.

Item libros sex.

Item caxectam unam de coreo munitam de argento.

Item facitergia quatuor roddichillata.

Item tobaliam unam tarasiatam de seta rubea et viridi.

Item coperchium unum de seta virgulatum.

Item superperchium unum de seta listata de auro.

Item cuxinum unum de panno de auro.

Item cuxinum aliud de panno de auro et billuto chilestro in medio.

Item caxectam unam de cristallo cum investa de ligno.

Item libros ecclesiasticos octo.

Marie.

Item caxectam unam de musia guarnitam de argento cum ymagine Virginis

Item certas reliquas sanctorum.

Item peciolum unum de zentini ${ }^{174}$.

\footnotetext{
${ }^{171}$ Così nel testo.

${ }^{172}$ Così nel testo.

${ }^{173}$ Per ysmaltata.

${ }^{174}$ Così nel testo.
} 
Item peciam unam in qua sunt picti duo agnus Dei.

Item peciolum unum de argento cum laqueo de seta viridi.

Item laqueum unum de seta viridi cum septem patenostris. una buleta.

Item marzapanum unum cum duobus purchillutis ${ }^{175}$ et cum filo de here et cum

Item arbolectum unum de perlis.

Item pecium unum de auro de seta.

Item pecium unum de cinchto viridi et de auro.

Item buxulam unam de ebore cum certis cartis scriptis.

Item taciam unam de argento.

Item burciam unam in qua sunt reliquie sanctorum.

Item pecium unum de burdo. tela viridi.

Item cultram unam seu paramurum de panno murisco de seta infoderatum de cindato viridi.

Item paramurum unum de panno de auro et billuto carmixino infoderatum de

Item calicem unum de argento deauratum cum pathena.

Item ampullucias duas de argento.

Item unam de argento.

Item campanellam unam de argento.

Item sichium unum de argento.

Item incinserium unum de argento.

Item navetam unam de argento.

Item candelabrum unum de argento.

Item ymaginem unam de albastro sancte Marie.

Item investam unam de billuto cum corporalibus.

Item palium unum de cindato rubeo infoderatum de tela viridi.

Item aliud palium de diaspro infoderatum de tela viridi.

Item palium aliud de panno aureo infoderatum de tela chilestra.

Item capam unam de diaspro infoderatam de cindato rubeo.

Item aliam capam de sita infoderatam de cindato rubeo.

Item capas duas de seta chilestra imbructatis de auro.

Item $^{176}$ cunixellam et diametricam de seta eiusdem panni.

Item conectam unam de musia.

Item infurras tres de tela cilestra de capis.

Item casubulam unam de seta.

Item pannum unum de altari de diaspro imbructatum de auro.

Item pannum unum de camuca de altari infoderatum de tela chilestra.

Item suppillicias quinque.

Item toballias de altari tres.

Item albam unam.

Item frontale de altari unum de seta.

Item stolas duas.

Item manupula duo.

Item admictum unum.

Item frontale aliud de panno de auro.

Item cuxinellum unum de seta.

\footnotetext{
${ }^{175}$ Così nel testo.

${ }^{176}$ Segue cur espunto.
} 
rubeo.

Item capam unam de panno de auro de presbitero infoderatam de cindato

Item linthiamina tria.

Item manticum unum firciatum de billuto et panno de auro.

Item mantum unum de seta infoderatum [c. $20 \mathrm{v}$.] de cindato ialino.

Item ciprensem unum et mantum ${ }^{177}$ de billuto chilestro stillatum de auro.

Item ciprensem de billuto violato cum arborectis de auro.

Item capam unam de ${ }^{178}$ cavalcari de billuto chilestro stillato de auro infoderatam de cindato rubeo.

Item mantum unum de billuto biridi cum frixo de auro ad arma de Aragona. cindato blevi.

Item manticum unum de seta murisca inbructato de auro, infoderatum de

Item bavarolum unum de panno de auro.

Item paramurum unum ferciatum de auro et billuto rubeo cum ferciis decem.

Item paramurum aliud ferciatum de auro et billuto rubeo cum ferciis novem. Item sambucas duas de argento cum frenis.

Item cuxinos duos de billuto et auro ad arma de Navarra.

Item cuxinum aliud de billuto et de auro.

Item cortinam unam de billuto rubeo ${ }^{179}$ et panno de auro ferciatam.

Item supracelum ${ }^{180}$ unum ferciatum de billuto rubeo et de auro.

Item cuxinum unum de panno murisco.

Item cortinam unam de panno murisco.

Item picteram unam de perlis consistentem in arborectis quatuordecim.

Item imperlatellas duas.

LXXXIIII ${ }^{\text {or }}$

Item imperlatellam unam ad decem postas cum zafiris decem et perlis

Item aliam imperlatellam cum partitorio cum rosetis de argento.

Item aliam imperlatellam cum perlis CXVI.

CVII.

Item aliam imperlatellam cum quatuor zefiris et quatuor balaxis ${ }^{181}$ et perlis

Item canactam unam ad decem posta ${ }^{182}$ cum quatuor zafiris.

Item par unum de paternostris cum perlis LXXXXVI, et quatuor zefiris et

tribus balaxis et XIIII buctonis de argento.

Item imperlatellam aliam cum perlis CXXV et zafiris VI et quatuor balaxis.

Item par unum auricularium cum certis lapidibus et perlis.

Item burzam unam laboratam ad acum.

Item buctonos de perlis XXXXVIII.

Item mergulum unum de corona cum ysmaltis sex.

Item pecium unum de iorlanda de auro cum uno ysmaraldo. duodecim.

Item duodecim pecia de iorlanda de auro cum duobus perlis et petris

Item novem pecia de iorlanda cum octo lapidibus et perlis XXXVI.

Item dintiglerium unum de argento.

\footnotetext{
${ }^{177}$ Segue et espunto.

${ }^{178}$ Segue cali espunto.

${ }^{179}$ Segue cum ferciis decem espunto.

${ }^{180}$ Segue aliud espunto.

${ }^{181}$ Segue Item par unum auricularium espunto.

${ }^{182}$ Così nel testo.
} 
Item muscam unam de argento deauratam cum certis lapidibus et perlis. Item scrimarolos tres de ebore.

Item pectinos de ebore duos.

Item pectinem unum de buxio.

Item buxulam unam de ebore.

Item brucchetam unam de curallo.

Item buctonos de argento undecim.

Item buctonos quatuor de curallo.

Item buctonos alios quatuor de argento.

Item medium cuxinellum de auro imburductum de auro.

Item capucheum unum cathalaniscum de panno de auro.

Item arborectos duos de curallo.

Item cocharellas duas de argento deauratas.

Item cocharellas duas de cristaldo fractas.

Item flasconetum unum de argento.

Item duos agnus Dei de argento deauratos.

Item buxulectam unam de argento cum crucetha in superius.

Item calamarium unum de argento muriscum.

Item in quadam pecia duos zaffiros, unum balaxium, unum ysmaraldum parvum et tres peciolas de argento.

Item buctonos de ambra $\mathrm{XX}^{\mathrm{ti}}$ septem.

Item cocharellam unam de argento.

Item in quadam pecia lapides alique ingastate.

Item gruppum unum cum certis peciis de argento.

Item partituri de paternostris de argento deaurato in quadam gruppo.

Item lapides ingastati in auro undecim, videlicet balaxos quinque magnos et

tres parvos, tres zaffiros.

Item decem lapides infilatos.

Item decem et octo [c. $21 \mathrm{r}$.] lapides ingastatos in auro.

Item mergulos de corona tres.

Item caxectam unam deauratam.

Item quendam hominem silvestrem de argento deauratum, cum cornu in ore

et quadam targa in pede in una vesta.

Item signaculum unum de argento imperlatum et cum petris.

Item maraxiam unam de cristallo in una vesta.

Item picteriam unam de perlis consistentem in $\mathrm{XX}^{\mathrm{ti}}$ peciis, videlicet undecim

magnis et novem parvis.

Item cayulam unam de perlis cum cannolectis de auro.

Item pectinem unum de ebore.

Item in uno gruppo certos lapides et perlas.

Item caxictam unam de coreo.

Item conectam unam.

Item certos cannolectos de cayula.

Item duos anulos sine lapidibus et tres cum lapidibus.

Item unum agnus Dei.

Item reliquas certas ${ }^{183}$ in una conecta inclusas.

Item duas arbores de curallo cum intornis serpe XIIII.

\footnotetext{
${ }^{183}$ Segue cum cannolectis de auro espunto.
} 
Item scalam unam de argento ad arma Sicilie, de Aragona et de Navarra cum sua investa.

Item caxectam unam cum certis rebus.

Item ciprensem unum et mantellum unum de billuto carmixino.

Item cortinam unam de seta biridi et rubea consistentem in pecias quatuor.

Item cultram unam muriscam infoderatam de tela viridi.

Item bunectos duos de panno rubeo.

Item cuxinos duos de billuto laceratos ad arma de Navarra.

cindato rubeo.

Item manticum unum de seta alba imbructatum de auro et infoderatum de

Item copertorium de lecto de skinis de vayris.

Item copertorium aliud de armenis.

Item tunicam unam veterem de panno de auro.

Item pallium unum de altari de seta infoderatum de tela chilestra.

Item albam unam de presbitero.

Item infurram de panno de seta rubea.

Item mensale unum.

Item cuxinum unum muriscum.

Item chilonam unam ialinam.

Item cortinam unam et imborlachium de panno rubeo consistentem in quinque

peciis.

Item cultram unam de panno de auro infoderatam de cindato rubeo.

Item mataracia tria de cindato rubeo et ialino plena bonbice.

Et ideo renunciando excepcioni rerum predictarum non habitarum et non receptarum per modum predictum et excepcioni doli, mali et omni alii iuri racioni et consuetudini, facimus vobis de predictis presentem apocham de recepto. Et cum a bonis predictis defficiant que casualiter amissa fuerunt quadam caxecta parvissima eboris argento munita unum pari linthiaminum panni lini et quedam pecia cortine panni virmilii, eadem vobis relaxamus et remittimus graciose et nullam volumus vobis exinde fieri questionem. munitam.

In quorum testimonium hanc fieri iussimus sigillo nostro ducis predicti

Datum et actum Cathanie vicesima octava die februarii prime indicionis anno Dominice incarnacionis MCCCXCII, regnique nostri dicti regis primo et dicte regine XVI.

Lo duch firmamus.

Signa Martini et Marie etc. et infantis Martini etc. qui hec concedimus et

Testes sunt qui ad predicta presentes fuerunt, nobilis Huguetus de Sancta Paçe camerlengus [c. 21 v.] et Poncius Dentença maiordomus milites consiliarii domini ducis predicti.

Signum Guillelmi Poncii secretarii domini ducis Montisalbi predicti, auctoritateque regali et reginali puplici notarii per totum regnum Sicilie, qui de predicti domini ducis mandato premissis interfuit, eaque scribi fecit et clausit cum rasis in LIII linea, LXXXXVI et LIIII linea perlis XXXVIII.

Dominus dux mandavit mihi Guillemo Poncii in cuius posse firmavit. 